Draft version August 8, 2018

Preprint typeset using $\mathrm{LAT}_{\mathrm{E}} \mathrm{X}$ style emulateapj v. 5/2/11

\title{
MORPHOLOGY AND KINEMATICS OF WARM MOLECULAR GAS IN THE NUCLEAR REGION OF ARP 220 AS REVEALED BY ALMA
}

\author{
Naseem Rangwala ${ }^{1,2}$, Philip R. Maloney ${ }^{1}$, Christine D. Wilson ${ }^{3}$, Jason Glenn ${ }^{1}$, Julia Kamenetzky ${ }^{1}$, And Luigi \\ SPINOGLIO ${ }^{4}$ \\ Draft version August 8, 2018
}

\begin{abstract}
We present Atacama Large Millimeter Array (ALMA) Cycle-0 observations of the CO $J=6-5$ line in the advanced galaxy merger Arp 220. This line traces warm molecular gas, which dominates the total CO luminosity. The CO emission from the two nuclei is well resolved by the $0.39^{\prime \prime} \times 0.22^{\prime \prime}$ beam and the exceptional sensitivity and spatial/spectral resolution reveal new complex features in the morphology and kinematics of the warm gas. The line profiles are asymmetric between the red and blue sides of the nuclear disks and the peak of the line emission is offset from the peak of the continuum emission in both nuclei by about $100 \mathrm{pc}$ in the same direction. CO self-absorption is detected at the centers of both nuclei but it is much deeper in the eastern nucleus. We also clearly detect strong, highly redshifted $\mathrm{CO}$ absorption located near the southwest side of each nucleus. For the eastern nucleus, we reproduce the major line profile features with a simple kinematic model of a highly turbulent, rotating disk with a substantial line center optical depth and a large gradient in the excitation temperature. The red/blue asymmetries and line-to-continuum offset are likely produced by absorption of the blue (SW) sides of the two nuclei by blue-shifted, foreground molecular gas; the mass of the absorber is comparable to the nuclear warm gas mass $\left(\sim 10^{8} \mathrm{M}_{\odot}\right)$. We measure an unusually high $L_{\mathrm{CO}} / L_{\mathrm{FIR}}$ ratio in the eastern nucleus, suggesting there is an additional energy source, such as mechanical energy from shocks, present in this nucleus.
\end{abstract}

Subject headings: galaxies: ISM, galaxies: active, techniques: interferometric, galaxies: kinematics and dynamics

\section{INTRODUCTION}

As the closest example $\left(D=77 \mathrm{Mpc}, 1^{\prime \prime}=373 \mathrm{pc}\right)$ of an ultra-luminous infrared galaxy (ULIRG), Arp 220 has been studied intensively at a wide range of wavelengths. It is an advanced merger of two gas-rich galaxies with high dust optical depth $\left(\tau_{100 \mu m} \sim 5\right.$; Rangwala et al. $2011)$ and a large reservoir of molecular gas $\left(\sim 10^{10}\right.$ $\mathrm{M}_{\odot}$ ) concentrated in its nuclear region (Scoville et al. 1997). It hosts one of the most extreme star-forming environments in the local universe and is a widely used template for interpreting observations of high- $z$, dusty star-forming galaxies. High-resolution ground-based radio interferometric observations show that it has two nuclei separated by roughly $1^{\prime \prime}$ (e.g., Scoville et al. 1997; Sakamoto et al. 2009), with evidence of a highly dustobscured AGN in its western nucleus (Downes \& Eckart 2007; Engel et al. 2011). Additionally, a kiloparsec-scale central molecular disk surrounding the two nuclear disks has been inferred from $\mathrm{CO} J=2-1$ interferometric observations by Scoville et al. (1997).

Observations of high- $J$ CO rotational transitions from the Herschel Space Observatory of nearby galaxies (including Arp 220) and Galactic star-forming regions have determined that there are generically two com-

\footnotetext{
${ }^{1}$ Center for Astrophysics and Space Astronomy, University of Colorado, 1255 38th street, Boulder, CO 80303

${ }^{2}$ Visiting Scientist, Space Science and Astrobiology Division, NASA Ames Research Center, Moffet Field, CA 94035

${ }^{3}$ Dept. of Physics \& Astronomy, McMaster University, Hamilton, Ontario, L8S 4M1, Canada

${ }^{4}$ Istituto di Astrofisica e Planetologia Spaziali, INAF, Via Fosso del Cavaliere 100, I-00133 Roma, Italy
}

ponents in the molecular gas: a warm (high-pressure) component traced by mid-to-high- $J$ lines that comprises a minority of the mass $(\sim 10 \%$ of the total molecular gas mass) but dominates the CO luminosity ( $\sim 90 \%$ of the total luminosity), and a cold (low-pressure) component traced by the lowest- $J$ lines, which dominates the mass (e.g., Panuzzo et al. 2010; Rangwala et al. 2011; Kamenetzky et al. 2012; Spinoglio et al. 2012; Meijerink et al. 2013; Goicoechea et al. 2013; Etxaluze et al. 2013; Pereira-Santaella et al. 2013; Papadopoulos et al. 2014). Comparison of the observed CO spectral line energy distributions (SLEDs) and far-infrared luminosities to theoretical models indicates that in several prominent cases (e.g., Arp 220, M 82, NGC 6240, M83) the gas heating is dominated by some mechanism other than UV or X-ray photons (as in photon-dominated and X-ray dominated regions) or cosmic rays; mechanical energy from shocks generated by supernovae and stellar winds are the most plausible sources. A recent study by Greve et al. (2014) using $\mathrm{CO}$ measurements in 62 local (U)LIRGs and 35 high- $z$ galaxies also concludes that mechanical energy is the more likely source of energy for this warm component. The pressure in the warm component is found to be typically two orders of magnitude larger than the cool component pressure (e.g., $10^{7} \mathrm{~K} \mathrm{~cm}^{-3}$ vs. $10^{5} \mathrm{~K} \mathrm{~cm}^{-3}$ ). These findings suggest that this warm component of the molecular gas is intimately associated with the feedback processes, which play an essential role in galaxy formation and evolution.

Our ongoing Herschel archival survey is systematically deriving physical conditions of the molecular gas in a 
large sample $(\sim 200)$ of nearby galaxies by modeling the combined observations from the Herschel SPIRE-FTS instrument, which provides CO SLEDs from $J=4-3$ to $J=13-12$, and ground-based measurements of low$J$ CO lines. The results from the analysis of an initial sample of 17 galaxies (Kamenetzky et al. 2014) confirmed the main findings described above. This study also found that the mid- $J$ CO transitions $(J>4-3)$, where the CO spectral line energy distribution peaks, primarily arise in the warm component and their line luminosities are well correlated with the total CO luminosity. Thus these transitions can be used as a reliable tracer of warm molecular emission. Because of its relatively large beams, Herschel could not spatially resolve most galaxies, so only average properties of the warm gas have been inferred, and it has been difficult to associate the warm gas with the distribution of star formation, cool molecular gas, and nuclear activity.

The CO $J=6-5$ line is now accessible with ALMA, which can finely resolve the morphology and kinematics of the warm gas in many nearby galaxies. This transition has been previously observed in Arp 220 with the SMA (Matsushita et al. 2009). However, the lesser resolution and lower sensitivity of SMA at these higher frequencies could not resolve the emission from the two nuclei. In this paper, we present the first high-resolution and high-S/N maps of the warm molecular gas in Arp 220 using the CO $J=6-5$ line. The corresponding continuum observations (at $435 \mu \mathrm{m})$ were presented in Wilson et al. (2014, Paper I hereafter). These observations showed that the western nucleus is considerably warmer and more optically thick $\left(T_{\text {dust }}=200 \mathrm{~K}, \tau_{434 \mu \mathrm{m}}=5.3\right)$ than the eastern nucleus $\left(T_{\text {dust }}=80 \mathrm{~K}, \tau_{434 \mu \mathrm{m}}=1.7\right)$. The two nuclei combined account for roughly $80 \%$ of the total infrared luminosity of Arp 220.

The ALMA observations and data reduction are presented in Section 2. The observed morphology and kinematics in the CO $J=6-5$ emission is described in Section 3 followed by detailed modeling of the kinematics of the nuclear disks in Section 4. We discuss disk stability and the energetics of the warm molecular gas in Sections 5 and 6 , respectively. Detection of absorption in the $\mathrm{SiO} J=16-15$ is presented in Section 7 and our conclusions are summarized in Section 8.

\section{OBSERVATIONS AND DATA REDUCTION}

Arp 220 was observed with ALMA on 2012 December 31 (Project 2011.0.00403.S) at the end of cycle-0 in an extended/hybrid configuration. The Band 9 receivers were tuned to cover the $\mathrm{CO} \mathrm{J}=6-5$ transition (rest frequency 691.473 GHz; Arp 220's redshift $=0.01813$ ) and adjacent continuum, with a total bandwidth of $8 \mathrm{GHz}$; however, the usable bandwidth was somewhat reduced due to overlap between the spectral windows covering the CO line and the need to flag a handful of end channels in each of the four spectral windows. The observations covered projected baselines from 13 to $374 \mathrm{~m}$. The correlator was configured in low-resolution wide-bandwidth TDM mode to give a spectral resolution of $15.625 \mathrm{MHz}$. The total on-source integration time was 44.4 minutes using 23 antennas.

The data were flagged, calibrated, and imaged by ALMA staff prior to delivery. The data have two unusual technical problems. First, no absolute flux calibrator was observed as part of the data set. The absolute flux scale was set using a flux of $6.0 \mathrm{Jy}$ for the bandpass calibrator, 3C279, determined by a Band 9 observation taken within one day of these observations. This process introduces some additional uncertainty on the absolute flux scale which is difficult to quantify. Second, the signal-to-noise ratio on the phase calibrators is very low, which required a major adjustment to the standard cycle-0 calibration process. The process used by ALMA staff was as follows. (1) Obtain a bandpass calibration solution from the bandpass calibrator. (2) Use the bandpass calibrator to solve for phase offsets among the four spectral windows and two polarizations. (3) Obtain a solution for phase versus time from the bandpass and two gain calibrators while combining the data for both polarizations and all four spectral windows together. (4) Obtain a solution for amplitude versus time from the three calibrators again combining the data from all polarizations and spectral windows. Use this solution to estimate the fluxes $(0.32$, $0.40 \mathrm{Jy}$ ) of the two secondary calibrators. (5) Obtain a final solution for amplitude versus time using the adopted flux of the two gain calibrators.

For our analysis, we began with the delivered calibrated $u v$ data and made new, cleaned data cubes following the same steps carried out by ALMA staff using CASA version 4.1.0. Repeating the imaging allowed us to adjust the clean boxes to reduce the level of artifacts in the final cubes even further from the very good images originally delivered to us and to improve the resolution by switching to uniform weighting. We also imaged all four spectral windows and adjusted the velocity ranges used to make the continuum images (Wilson et al. 2014) to avoid all emission and absorption features.

The reduction notes from the ALMA staff noted that self-calibration was required to get the best images. Following their method, we carried out two rounds of phaseonly self-calibration using natural weighting and a relatively small clean box to include emission from the bright central region. A final round of self-calibration on both amplitude and phase significantly improved the residuals in the data cube. As noted by ALMA staff, we see significant amplitude corrections are required and these corrections correlate among the different spectral windows. These steps significantly improved the noise level in the cube, from $36 \mathrm{mJy} /$ beam to $16 \mathrm{mJy} /$ beam in the line free channels and from $100 \mathrm{mJy} /$ beam to $18 \mathrm{mJy} /$ beam in channels where the line emission was strong.

Before making the final image cube, we carried out continuum subtraction using a zeroth order fit to most of the channels in the two spectral windows located away from the bright $\mathrm{CO}$ emission. In the final imaging step, the clean box was adjusted with frequency to include all regions with significant emission, which improved the negative residuals in certain channels with bright emission. We made image cubes with both natural and uniform weighting to maximize sensitivity and angular resolution, respectively. We used a velocity resolution of $10 \mathrm{~km} \mathrm{~s}^{-1}$ for the CO data cube. As a final step, the uniform cube was smoothed slightly so that all channels would have the same beam $\left(0.39^{\prime \prime} \times 0.22^{\prime \prime}\right)$ at position angle $28^{\circ}$. The typical $1 \sigma$ noise in the cleaned uniform cube is $30 \mathrm{mJy} \mathrm{beam}^{-1}$ or $0.9 \mathrm{~K}$ per channel.

After a small correction for primary beam attenuation, the total integrated CO $J=6-5$ flux is 3670 


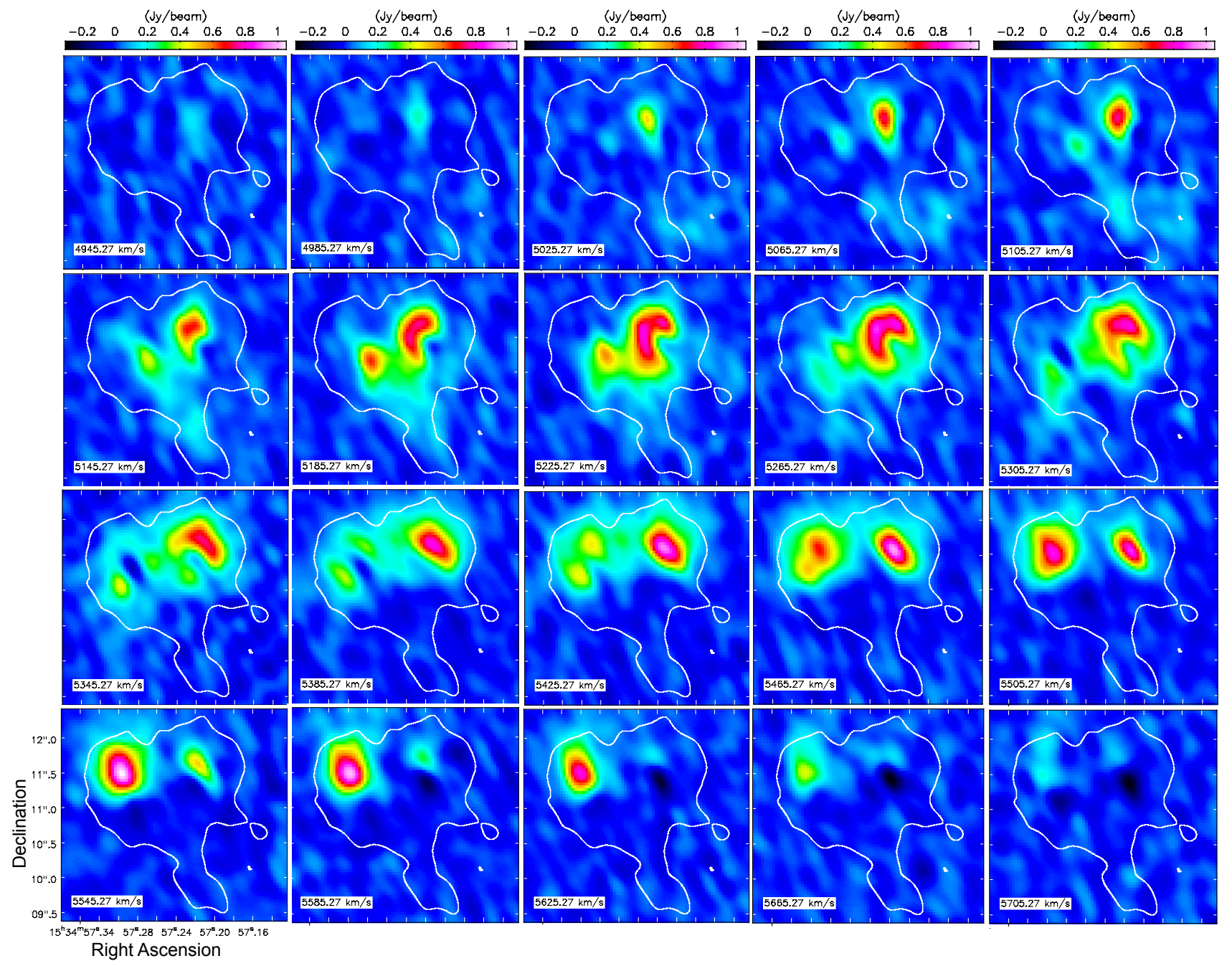

Figure 1. Channel maps covering the CO emission and absorption over $\sim 800 \mathrm{~km} \mathrm{~s}^{-1}$. The $1 \%$ contour level for the continuum emission is shown relative to the peak continuum flux of $1.38 \mathrm{Jy}_{\text {beam }}{ }^{-1}$. Redshifted CO absorption can be seen in the high velocity channels (5505 $\left.-5705 \mathrm{~km} \mathrm{~s}^{-1}\right)$. Note: The maps and spectra in this paper are not corrected for the small primary beam attenuation.

Jy $\mathrm{km} \mathrm{s}^{-1}$. This value is in agreement with the total flux obtained from Herschel-SPIRE of $4070 \pm 80 \mathrm{Jy}$ $\mathrm{km} \mathrm{s}^{-1}$ (Rangwala et al. 2011) within their calibration uncertainties (about 10\% for Herschel-SPIRE and 15\% for ALMA). Even in the absence of an absolute flux calibrator, the final flux calibration of our data cube is consistent with literature values.

The channel maps from the uniform data cube are presented in Figure 1 for CO emission over $\sim 800 \mathrm{~km} \mathrm{~s}^{-1}$. The extent of the continuum emission is indicated by the $1 \%$ contour level. Deep CO self-absorption is detected around the systemic velocity of the eastern nucleus that appears to go below zero. Also, redshifted CO absorption is seen in higher velocity channels. These features are seen in the spectra at the continuum positions even before the continuum is subtracted. There are gaps in the data cube over two velocity intervals: $4245-4305$ $\mathrm{km} \mathrm{s}^{-1}$ and $5735-6035 \mathrm{~km} \mathrm{~s}^{-1}$ (see Figure [3). The latter gap is close to the redshifted $\mathrm{CO}$ absorption and limits our ability to measure the full velocity extent of this absorption feature. These features are further discussed in $\S 3$.

\section{OBSERVED MORPHOLOGY AND KINEMATICS OF THE WARM MOLECULAR GAS}

The CO $J=6-5$ emission traces the warm molecular gas peaks at the centers of the eastern and western nuclei, which are unambiguously resolved in the $0.39^{\prime \prime} \times 0.22^{\prime \prime}$ resolution CO map (Figure [2a), and has an extended component surrounding the two nuclei. This overall morphology agrees with the $\mathrm{CO} J=2-1$ and $\mathrm{CO} J=3-2$ maps of Sakamoto et al. (1999, 2008), in which the emission associated with the two nuclei was characterized as compact nuclear disks that are embedded in an extended outer disk. A simple Gaussian fitting to the two nuclei shows that the western nucleus is almost circular with a deconvolved size (FWHM) of $\sim 0.55^{\prime \prime}$ (205 pc using $\left.1^{\prime \prime}=373 \mathrm{pc}\right)$, while the eastern nucleus is elongated in the NE-SW direction with a deconvolved size (FWHM) of $0.74^{\prime \prime} \times 0.59^{\prime \prime}(276 \mathrm{pc} \times 220 \mathrm{pc})$. This indicates that the $\mathrm{CO}$ emission is extended compared to the dust continuum emission, for which the half-maximum diameters are $76 \times(\leq 70) \mathrm{pc}$ and $123 \times 79 \mathrm{pc}$ for the western and eastern nucleus, respectively (Paper I). However, there is likely extended, low surface brightness dust emission that is below the current sensitivity of our observations: the 

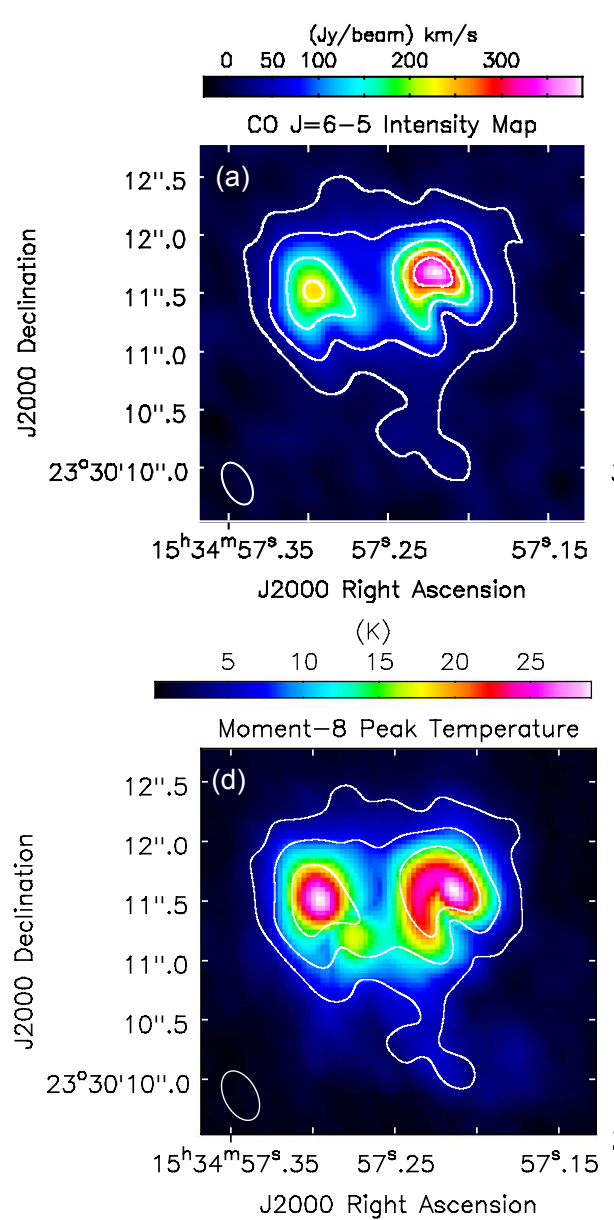
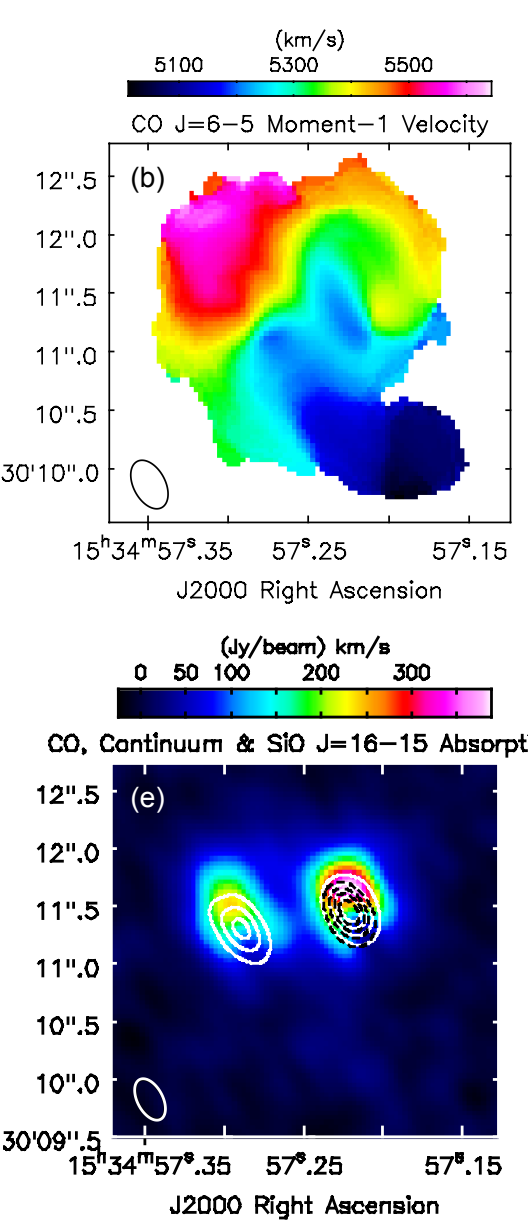

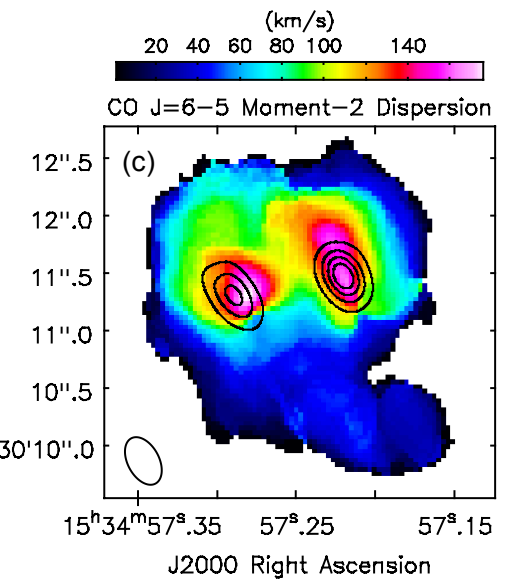

(Jy/beam) $\mathrm{km} / \mathrm{s}$ $\begin{array}{lllll}0 & 0.2 & 0.4 & 0.6 & 0.8\end{array}$

CO $\mathrm{J}=6-5$ Emission and Absorption

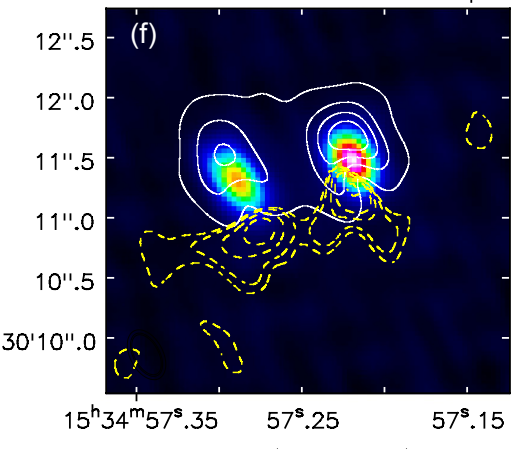

J2000 Right Ascension

Figure 2. ALMA high-resolution images of the CO $J=6-5$ line and $691 \mathrm{GHz}$ continuum in Arp 220. (a) CO integrated intensity (moment-0) map made from uniform cube. The $10 \%, 20 \%, 40 \%, 60 \%$ and $80 \%$ contour levels are shown relative to the peak flux of 389 Jy beam ${ }^{-1} \mathrm{~km} \mathrm{~s}^{-1}$ in the western nucleus. (b) Mean velocity Field (moment-1). (c) Velocity dispersion (moment-2) map. The 20\%, 40\%, $60 \%$ and $80 \%$ continuum contours are shown relative to the peak continuum flux of $1.15 \mathrm{Jy} \mathrm{beam}^{-1}$ in the western nucleus. (d) Peak temperature (moment-8) map and integrated CO intensity contours in White. (e) The peak of the line emission (color map) is offset from the peak of the continuum emission (solid contours) by roughly 100pc. The SiO $J=16-15$ line absorption (dashed contours $-20 \%, 40 \%$, $60 \%$ and $80 \%$ of $-70 \mathrm{Jy}_{\text {beam }}^{-1} \mathrm{~km} \mathrm{~s}^{-1}$ ) is detected only in the western nucleus and is unresolved. (f) Moment-0 contours of CO $J=6-5$ redshifted absorption over $5505-5725 \mathrm{~km} \mathrm{~s}^{-1}$ (yellow contours). The continuum emission (color) and line emission (solid contours) are overplotted. The yellow contour levels are $20 \%, 30 \%, 50 \%, 70 \%$ and $90 \%$ relative to $-25 \mathrm{Jy}_{\text {beam }}^{-1} \mathrm{~km} \mathrm{~s}^{-1}$. The contours depict both the compact and extended components of the redshifted absorption.

combined continuum emission at $692 \mathrm{GHz}$ detected in the two nuclei is about $80 \%$ of the total continuum emission measured in the ALMA map (Paper I) and only 56\% of the total continuum emission measured in the Herschel data (Rangwala et al. 2011). The peak flux, integrated flux and source sizes for $\mathrm{CO} J=6-5$ emission are listed in Table 1.

The overall $\mathrm{CO} J=6-5$ velocity field (Figure 2 $\mathrm{b}$ ) is also similar to the CO $J=2-1$ and CO $J=3-2$ maps, which show a large velocity gradient in the NE SW direction. The velocity extent is $\sim 500 \mathrm{~km} \mathrm{~s}^{-1}$ within $0.3^{\prime \prime}$ of the nuclei, again similar to the $\mathrm{CO} J=2-1$ velocity extent (Sakamoto et al. 1999). The CO $J=6-5$ velocity dispersion $(\sigma=\mathrm{FWHM} / 2.3548)$ map (Figure 2 (c)) shows the dispersion peaks are coincident with the continuum peaks, with values as large as $175 \mathrm{~km} \mathrm{~s}^{-1}$ and $160 \mathrm{~km} \mathrm{~s}^{-1}$ for the eastern and western nucleus, respectively.

To compare with the $\mathrm{CO} J=2-1$ and $\mathrm{CO} J=3-2$ maps from Sakamoto et al. (2008), which have a beam FWHM of $0.5^{\prime \prime}$, we convolved the CO $J=6-5$ line map to match their beam size. Using these maps we find that about $50 \%$ of the total detected CO $J=6-5$ emission arises from within the nuclear region (i.e., within a radius of $\left.0.45^{\prime \prime}\right)$. This fraction is higher than for the low- $J$ lines $(\sim 30 \%)$, implying that the warm molecular emission is relatively more compact.

The peak temperature (moment-8) map, Figure 2(d), displays two noticeable features in comparison with the integrated flux map: the semicircular ridge of emission in the western nucleus is more prominent in peak temperature than in integrated flux, and there is an off-nucleus "hot spot" in the eastern nucleus (although its temperature is lower than the nuclear peak). Comparable highresolution maps in additional $\mathrm{CO}$ transitions will be required to determine whether these temperature features correspond to variations in excitation temperature.

Overplotting the continuum contours on the CO $J=$ $6-5$ integrated intensity map (Figure 2 e) shows an offset between the peak of the continuum emission and the peak of the line emission in both nuclei; this offset is about $0.2^{\prime \prime}$ and has the same direction. This offset is not seen in the 
Table 1

ALMA Arp 220 line Measurements

\begin{tabular}{|c|c|c|c|}
\hline Quantity & Eastern Nucleus & Western Nucleus & Units \\
\hline \multicolumn{4}{|c|}{$\mathrm{CO} J=6-5$ Emission } \\
\hline $\begin{array}{l}\text { Peak flux location } \\
\text { Integrated flux }\left(1^{\prime \prime} \times 1^{\prime \prime}\right) \\
\text { Peak flux } \\
\text { Gaussian FWHM } \\
\text { Gaussian-fit Integrated flux } \\
\text { Line-to-Continuum ratio } \\
L_{\mathrm{CO}} / L_{\mathrm{FIR}}{ }^{\mathrm{a}} \\
\text { Deconvolved FWHM } \\
\text { Warm Gas Mass }{ }^{\mathrm{b}} \\
\text { Warm Gas Column Density }^{\mathrm{c}}\end{array}$ & $\begin{array}{l}(15: 34: 57.30,+23: 30: 11.53) \\
1070 \\
330 \\
0.83 \times 0.63 \\
1565 \\
1035 \\
6.7_{-4.4}^{+16} \times 10^{-5} \\
0.74 \times 0.59 \\
1.4 \times 10^{8} \\
9.2 \times 10^{22}\end{array}$ & $\begin{array}{l}(15: 34: 57.22,+23: 30: 11.68) \\
1305 \\
515 \\
0.66 \times 0.63 \\
1795 \\
990 \\
6.0_{-3.6}^{+29} \times 10^{-6} \\
0.59 \times 0.54 \\
1.6 \times 10^{8} \\
1.5 \times 10^{23}\end{array}$ & $\begin{array}{l}\text { J2000 } \\
\mathrm{Jy} \mathrm{km} \mathrm{s}^{-1} \\
\mathrm{Jy} \mathrm{beam}^{-1} \mathrm{~km} \mathrm{~s}^{-1} \\
\mathrm{Jy} \mathrm{km} \mathrm{s}^{-1} \\
\mathrm{~km} \mathrm{~s}^{-1} \\
\prime \prime \\
M_{\odot} \\
\mathrm{H} / \mathrm{cm}^{-2}\end{array}$ \\
\hline \multicolumn{4}{|c|}{$\mathrm{SiO} \mathrm{J}=16-15$ Absorption } \\
\hline $\begin{array}{l}\text { Peak flux location } \\
\text { Peak flux } \\
\text { Gaussian FWHM, PA } \\
\text { Gaussian-Fit Integrated flux } \\
\text { Mean depth } \\
\text { Velocity extent }\end{array}$ & $\begin{array}{l}\text { No Detection } \\
- \\
- \\
- \\
- \\
-\end{array}$ & $\begin{array}{l}(15: 34: 57.22,+23: 30: 11.43) \\
-80 \\
0.385 \times 0.255,27 \\
-87 \\
-0.23 \\
\gtrsim 400\end{array}$ & $\begin{array}{l}\text { J2000 } \\
\text { Jy beam }{ }^{-1} \mathrm{~km} \mathrm{~s}^{-1} \\
\left({ }^{\prime \prime},{ }^{\circ}\right) \\
\text { Jy km s }^{-1} \\
\text { Jy beam }^{-1} \\
\mathrm{~km} \mathrm{~s}^{-1}\end{array}$ \\
\hline
\end{tabular}

Note. - The peak and integrated fluxes corrected for primary beam attenuation are reported from the natural weighted (higher sensitivity) moment-0 map with a beam size of $0.447^{\prime \prime} \times 0.273^{\prime \prime}\left(\mathrm{PA}=28^{\circ}\right)$, while the source FWHM is reported from the higher-resolution uniform weighted map with a beam size of $0.39^{\prime \prime} \times 0.22^{\prime \prime}$.

The $\mathrm{S} / \mathrm{N}$ in our data is very high - 55 in the peak flux and $>400$ on the integrated flux - leading to very small measurement errors; thus they are not reported. The overall uncertainties are dominated by systematic calibration uncertainties of $\sim 15 \%$.

${ }^{a}$ Using the $L_{\text {FIR }}$ measured from the ALMA $691 \mathrm{GHz}$ continuum emission in Paper I. Note that the large range in uncertainty in this ratio arises entirely from the large systematic uncertainty on the $L_{\text {FIR }}$.

${ }^{\mathrm{b}}$ Using $M\left(H_{2}\right)_{\text {warm }} / L_{C O 6-5}=21 \mathrm{M} \odot / \mathrm{L}_{\odot}$ derived from Herschel FTS CO SLED (Rangwala et al. 2011)

c Using the Gaussian FWHM and Area $=\pi R^{2}$

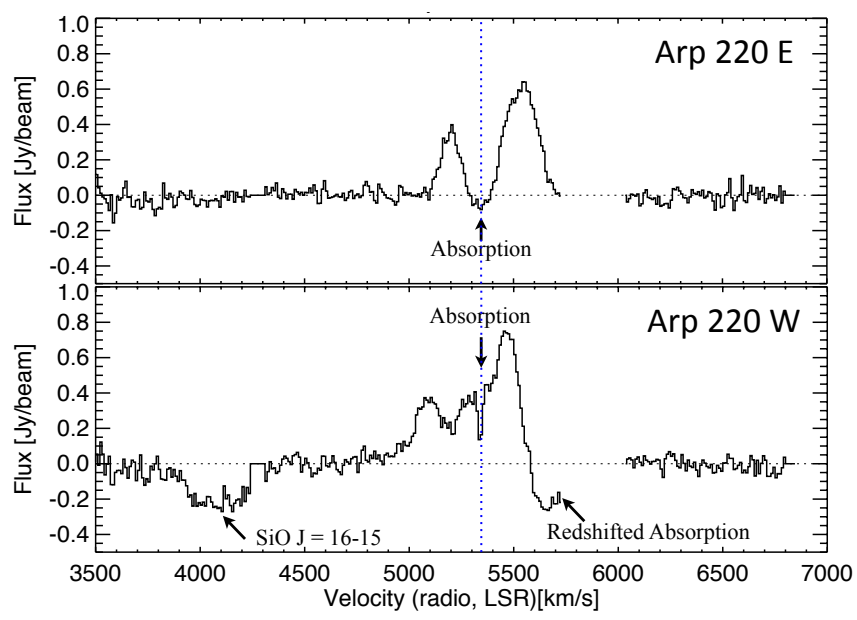

Figure 3. Continuum subtracted spectral line profiles at the location of the continuum peak for the eastern and western nuclei, in the reference frame of $\mathrm{CO}$.

low- $J$ lines (up to $J=3-2$ ) from previous observations. We explain in Section 4 that this offset is only apparent, and is likely caused by absorption of the entire SW side of the line emission in the two nuclei.

Moreover, $\mathrm{CO}$ absorption is detected at the centers of the two nuclei as shown in Figure 3 the absorption is much deeper in the eastern nucleus and goes below zero in the continuum subtracted spectra. This absorption is also shown in the channel map close to the systemic velocity of the eastern nucleus (Figure 1) channel 5345 $\mathrm{km} \mathrm{s}^{-1}$ ). The central dip seen in the eastern nucleus suggests the presence of absorption; that this dip goes below zero (these are continuum subtracted spectra) indicates both that this feature is the result of absorption and that we are seeing absorption of the $6-5$ line against the continuum source, i.e., the absorbing gas is absorbing both line and continuum emission.

The deep CO absorption at the center was not reported from the interferometric observations of the $\mathrm{CO} J=2-1$ and $3-2$ lines with $0.5^{\prime \prime}$ resolution (Sakamoto et al. 2008). In Figure 4, the CO $J=3-2$ profile from Sakamoto et al. (2009, private communication 2015), observed using a $0.3^{\prime \prime}$ beam, is overplotted on our $\mathrm{CO}$ $J=6-5$ profile (at the continuum peak). This comparison clearly shows that for the eastern nucleus a similar absorption dip is present in the low- $J$ CO line, although it was not discussed by Sakamoto et al. (2009) (further discussion is provided in section 4). In fact, the CO $J=3-2$ line shows a double-peaked profile in the eastern nucleus similar to that of $\mathrm{CO} J=6-5$, except the blue side is much weaker than the red side. Comparison of the western nucleus profiles also shows similarity between the two transitions, i.e., a central absorption dip and an overall complexity in the line profiles.

The spectrum of the western nucleus in Figure 3 (bottom panel) also shows redshifted $\mathrm{CO}$ absorption at velocities between roughly $5505 \mathrm{~km} \mathrm{~s}^{-1}$ and $5725 \mathrm{~km} \mathrm{~s}^{-1}$, much higher than the systemic velocity of the system. Absorption is also detected towards the eastern nucleus but in off-center positions and in higher velocity channels 
(see Figure1 (channel $5705 \mathrm{~km} \mathrm{~s}^{-1}$ ) and Figure 5). The redshifted absorption is shown with yellow contours in Figure 2(f), which also shows the CO emission in solid contours and the continuum emission in color. The absorption has a deep, compact component as well as a shallow, extended component. We note that although the shallow extended component is present over many velocity channels, the less than ideal calibration of the data and the low $\mathrm{S} / \mathrm{N}$ mean that we cannot be certain it is not an artifact of the cleaning process. The peak of the redshifted $\mathrm{CO}$ absorption is also offset in the two nuclei relative to the continuum peak but in the opposite direction compared to the line emission peak, i.e., towards the SW direction. Because this absorption is present towards both nuclei, its plausible that it arises from a continuous feature covering the southern part of the whole system, i.e., at least $400 \mathrm{pc}$ in length (roughly the distance between the two nuclei). This continuous redshifted absorption feature could be interpreted as an infalling molecular filament (see section 4.2), not unexpected in a chaotic late-stage merger. It is difficult to ascertain the full velocity extent of this feature because of gaps in our data but it is at least $200 \mathrm{~km} \mathrm{~s}^{-1}$ wide.

A highly excited $\mathrm{SiO}$ line, $J=16-15$, is detected in absorption (dashed contour in Figure 2(e); also see Figure [3), but only towards the western nucleus. This line is discussed further in Section 7.

In summary, the exceptional sensitivity and spectral resolution from ALMA has allowed us to detect several components in the warm molecular gas in Arp 220 showing more complexity in the morphology and nuclear kinematics than previously observed.

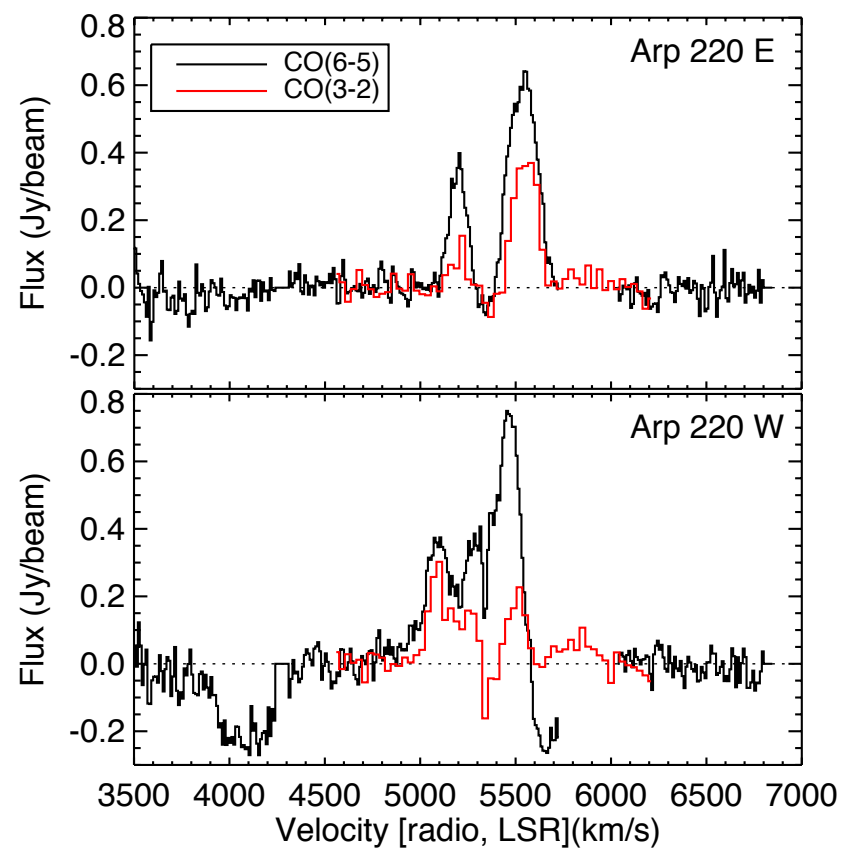

Figure 4. Comparison between the $\mathrm{CO} J=6-5$ (this work) and CO $J=3-2$ (Sakamoto et al. 2009, private communication $2015)$ velocity profiles at the location of the continuum peak for the eastern and western nuclei in $0.39^{\prime \prime} \times 0.22^{\prime \prime}$ and $0.3^{\prime \prime}$ beams, respectively.

\section{MODELING: KINEMATICS OF THE NUCLEAR DISKS}

Both the eastern and western nuclei show clear kinematic evidence for rotating disks. In addition to the velocity gradients clearly seen in Figure 2(b), in Figure 5 . we show spectra along the major axes of both nuclear disks, spaced at multiples of a half-beamwidth $(\sim 80 \mathrm{pc})$ from the dust continuum peaks. In both cases we see clear signs of rotation: a systematic shift in the velocity of the emission peak with position along the major axis, with an isolated red or blue peak on opposite sides of the continuum peak. The kinematics of the eastern nucleus, in particular, appear relatively simple, with a doublepeaked (but asymmetric) profile at the continuum center that shifts to single red- or blue-shifted peaks with increasing distance along the major axis. In comparison, the velocity profiles of the western nucleus are more complex. For this reason we present here a simple toy model intended to explain the main features of the spectra for the eastern nuclear disk, and then discuss the implications for both nuclei and the larger-scale gas distribution around the nuclei.

In the presence of velocity dispersion and systematic motion (e.g., rotation or outflow), we can write the line optical depth as a function of frequency as

$$
\tau_{\nu}=\tau_{0} \phi_{\nu}
$$

where $\tau_{0}$ is the line center optical depth and the Doppler line profile (for non-relativistic velocities) is given by

$$
\phi_{\nu}=\exp \left(-\left[\frac{\nu-\nu_{0}}{\Delta \nu_{D}}-\frac{\left(\nu_{0} / c\right) \mathbf{n} \cdot \mathbf{v}}{\Delta \nu_{D}}\right]^{2}\right)
$$

where $\Delta \nu_{D}$ is the Doppler width $(\sqrt{2}$ times the dispersion), $\nu_{0}$ is the line center frequency, $\mathbf{n}$ is the direction of propagation, and $\mathbf{v}$ is the velocity (e.g., Hummer \& Rybicki 1968).

The nuclear disk is substantially inclined to our line of sight, with $i \approx 50^{\circ}$ based on the deconvolved continuum source size (Paper I). For simplicity, we assume that the disk is perfectly edge-on. We also assume that the disk is uniform in the vertical direction, which means that the disk thickness is only a meaningless scaling parameter. This reduces the model to two dimensions, which considerably speeds up the numerical modeling. We expect that the inferred model parameters would only change by factors of order unity in going from this edge-on, 2D disk to a tilted, 3D disk, e.g., the true disk rotation curve will increase by $1 / \cos i$ from the model curve. The only features not captured by this approximation would be systematic motions that are confined largely or entirely to the disk vertical direction. While our simple edge-on model can only include features that are a function of projected distance from the disk center with no dependence on angle within or height above the disk, the match between the model spectra and the data are sufficiently good (see 4.2) that features violating this restriction are not required to explain the observed spectra.

We choose a coordinate system such that the $x$-axis lies in the plane of the sky, the $y$-axis is now the disk vertical direction, and the $z$-axis lies in the disk and is oriented along the line of sight towards the observer, with $\theta$ the angle in the disk with respect to the $x$-axis ( $\operatorname{so} \theta=\pi / 2$ points towards the observer). The radial coordinate $r$ then lies in the plane of the disk, and the distinction be- 
tween cylindrical and spherical radius has been removed (e.g., a spherical outflow lies purely in the disk plane in this approximation). The projected line-of-sight velocity is then

$$
\mathbf{n} \cdot \mathbf{v}=V_{c}(r) \cos \theta+V_{\text {out }}(r) \sin \theta
$$

for circular rotation velocity $V_{c}$ and purely radial outflow velocity $V_{\text {out }}$. The distortion of the line profile function by the systematic velocity terms plays a key role in determining the appearance of the disk emission. Note that equation (2) with equation (3) is antisymmetric about $\theta=\pi / 2$ in the rotation-only case, and symmetric in the outflow-only case (provided that the outflow is itself symmetric about this line), which cuts the needed amount of computation in half. However, it is neither in the case that both rotation and outflow are present.

We specify the line and continuum source functions and optical depths as a function of radius in the disk, which is assumed to be circular and axisymmetric. Both the source functions and the optical depth profiles are assumed to be Gaussians in radius $r$. With both line and continuum emission and absorption, the source function $S_{\nu}$ is given by the ratio of the combined emissivities $j_{\nu}$ to the sum of the absorption coefficients $\alpha_{\nu}$ :

$$
S_{\nu}=\frac{j_{\nu, l}+j_{\nu, d}}{\alpha_{\nu, l}+\alpha_{\nu, d}}
$$

with the $l$ and $d$ subscripts referring to line and dust, respectively. The optical depth at frequency $\nu$ is now

$$
\tau_{\nu}=\tau_{\nu, l}+\tau_{\nu, d} .
$$

In terms of the line-only and dust-only source functions, equation (4) can be written

$$
S_{\nu}=\frac{\alpha_{\nu, l} S_{\nu, l}+\alpha_{\nu, d} S_{\nu, d}}{\alpha_{\nu, l}+\alpha_{\nu, d}}
$$

i.e., the individual source functions weighted by their respective absorption coefficients, or, equivalently, by the corresponding values of $\tau_{\nu}$. Equations (44) and (6) can be simplified by noting that we can take the dust emissivity and absorption coefficient (and thus the dust source function) to be independent of frequency over the width of the line profile.

The emitted intensity along a line of sight through the disk is then

$$
I_{\nu}=\int_{z_{\min }}^{z_{\max }} S_{\nu} \exp \left[-\int_{z}^{z_{\max }} \alpha_{\nu}\left(z^{\prime}\right) d z^{\prime}\right] \alpha_{\nu} d z
$$

To solve this equation, we divide the disk into segments along the $z$-direction at each value of impact parameter $p$ (perpendicular distance from disk center); changing variables from distance along the line of sight to optical $\operatorname{depth} \tau_{\nu}(z)=\int_{z}^{z_{\max }} \alpha_{\nu}\left(z^{\prime}\right) d z^{\prime}, I_{\nu}$ is then the sum over pieces of the form

$$
S_{\nu}(z)\left(1-e^{-\tau_{\nu}(z)}\right) e^{-\tau_{\nu}^{t o t}(z)}
$$

5 Note that this is not a physical model, in the sense that the excitation of the $\mathrm{CO}$ line has not been calculated from conditions in the disk: the line source function and optical depth are specified independently of one another, and both the line and continuum source functions have arbitrary normalizations. This model is intended to aid in understanding the main features of the emission from the eastern nucleus, not reproduce it in detail. where $\tau_{\nu}(z)$ is the local optical depth, and $\tau_{\nu}^{\text {tot }}(z)$ is the total optical depth from depth $z$ to the surface of the disk. Hence

$$
I_{\nu}=\sum_{z_{\min }}^{z_{\max }} S_{\nu}(z)\left(1-e^{-\tau_{\nu}(z)}\right) e^{-\tau_{\nu}^{t o t}(z)}
$$

The model line profiles are calculated by convolving the disk emission with a gaussian beam, with the model beam size matched to the observations.

\subsection{Model Results - Axisymmetric rotating disk}

A reasonable match to the observed profiles for the eastern nucleus is provided by a model in which the disk has center-to-edge line center optical depth $\tau_{0, l}=4$, dust optical depth $\tau_{0, d}=1$, velocity dispersion $\sigma=85$ $\mathrm{km} \mathrm{s}^{-1}$, and the rotation curve is flat with projected circular velocity $V_{\mathrm{c}}=135 \mathrm{~km} \mathrm{~s}^{-1}$. The normalizations of the line and continuum source functions are arbitrary; the continuum source function and the line center optical depth have been adjusted to produce about the right levels for line and continuum for the disk center position and to match the absorption depth. In order to produce the deep central absorption the line center optical depth must be substantial $\left(\tau_{0, l}\right.$ at least $\sim$ a few $)$ and there must be a substantial gradient in the $\mathrm{CO} J=6-5$ line source function (i.e., the excitation temperature) as a function of radius, decreasing outward. Thus, the deep absorption at the center is primarily from self-absorption. However, flat rotation curve models generically predict that the line peaks for the center position $(p=0)$ lie closer to the systemic velocity than those for larger offsets, in disagreement with the observations. A substantial improvement results from including a velocity perturbation (or a bump) in the rotation curve in the inner $R \sim 100 \mathrm{pc}$ or so (compare the upper left panels of Figure 7 with the models shown in Appendix A). We have adopted the rotation curve shown in Figure 6, in which $V_{\mathrm{c}}$ peaks at about $240 \mathrm{~km} \mathrm{~s}^{-1}$. (The dip interior to the peak is just there to force $V=0$ at $R=0$; we have no sensitivity to emission (or its absence) on such small spatial scales.) The relative positions in velocity space of the red-wing peaks are in much better agreement with the observations in the model which includes the velocity perturbation shown in the upper left panel of Figure 7 than for the flat rotation curve model shown in Appendix A (upper left panel of Figure 15). The velocity bump also has the effect of broadening the line profile somewhat, which also improves the match with the data 6 We defer discussion of the observed rotation curve to $\S 4.4$.

The exact shape of the curve should not be taken too seriously, as the needed $V(R)$ depends on the spatial distribution of the CO line emissivity, and non-circular velocities may also play a role. It is evident, however, that the velocity perturbation needs to be quite significant $\left(\sim 100 \mathrm{~km} \mathrm{~s}^{-1}\right)$ - this is largely the result of the projected velocity $V_{\text {circ }}^{\text {proj }} \rightarrow 0$ as the impact parameter $p \rightarrow 0$. In the context of our models, it implies that there is a significant increase in the concentration of mass interior to $R \sim 100 \mathrm{pc}$.

6 Although it is more subtle, this also improves the match between the model and the data for the $\theta_{B} / 2$ offset positions, for the lower-amplitude peaks. 


\section{Eastern Nucleus - Major Axis}
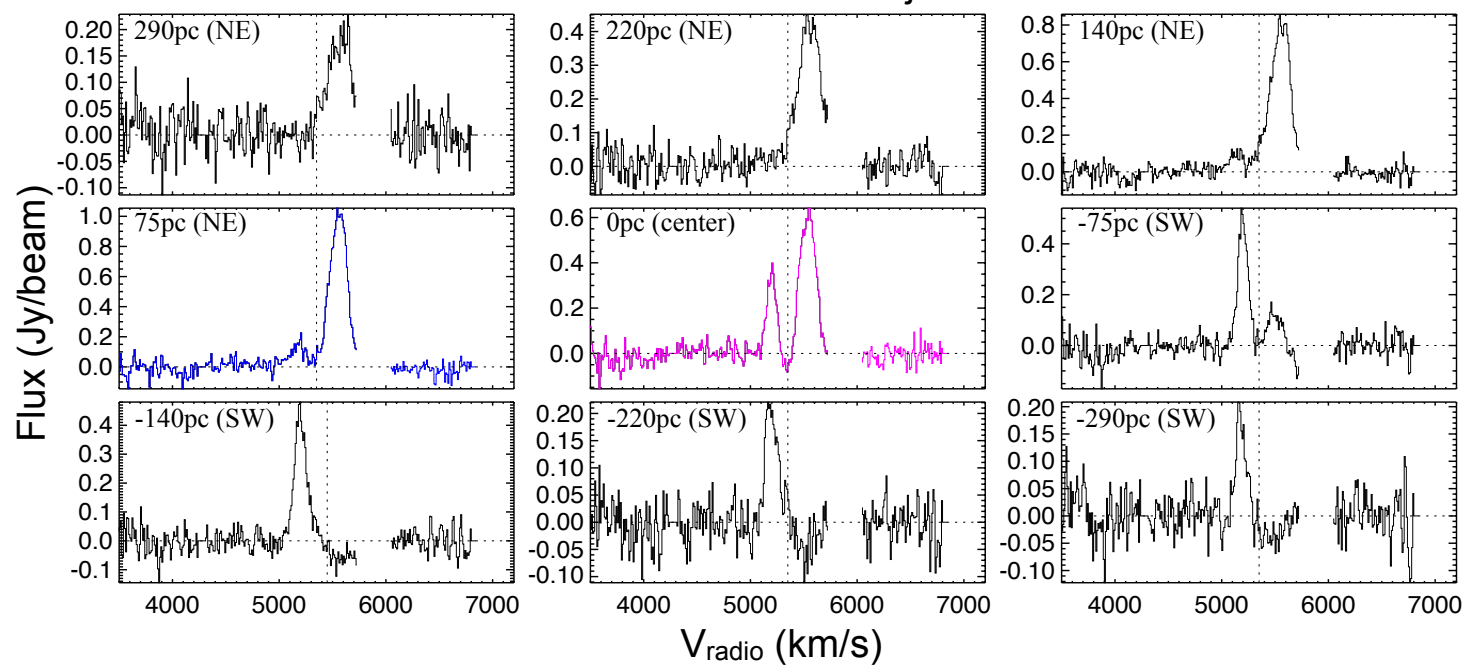

\section{Western Nucleus - Major Axis}
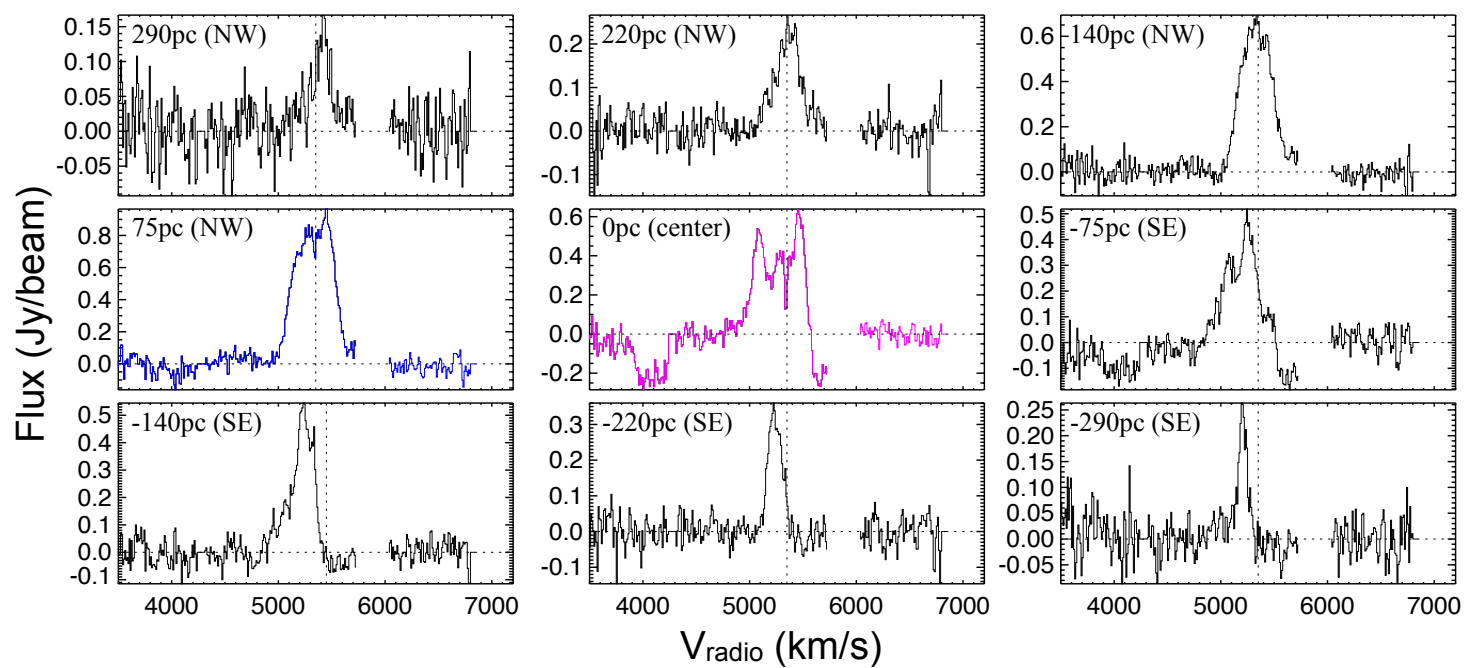

Figure 5. The velocity profiles along the major axes of the Eastern (for PA $\sim 35^{\circ}$ ) and Western $\left(\right.$ for PA $\sim 111^{\circ}$ ) nuclei. The blue and magenta colored profiles are at the location of the line peaks and continuum peaks, respectively. The vertical line marks the systemic velocity of the system at $5350 \mathrm{~km} \mathrm{~s}^{-1}$.

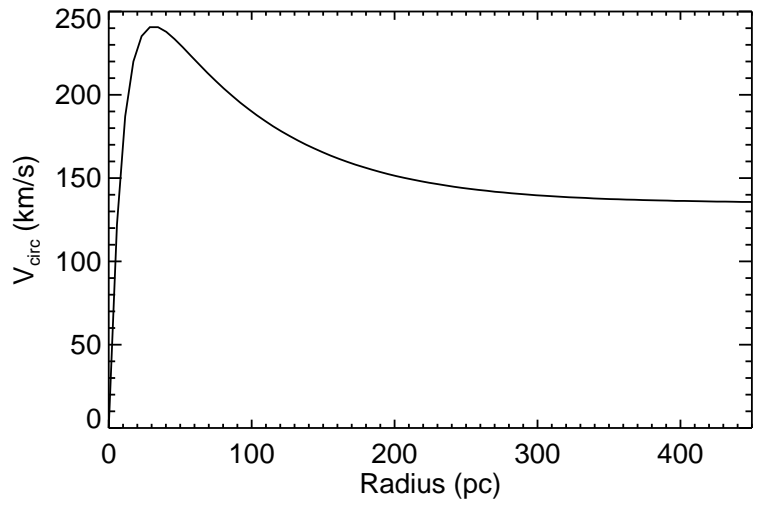

Figure 6. Rotation curve adopted in our kinematic modeling of the eastern nucleus. Note: The dip interior to the peak is just there to force $\mathrm{V}=0$ at $\mathrm{R}=0$; our data do not have sensitivity to emission (or its absence) on such small spatial scales.
The model and observed profiles are shown in Figure 7. The "red" and "blue" sides refer to which side of the double-sided profile dominates, so $V>0 \mathrm{~km} \mathrm{~s}^{-1}$ (model) or $V>5350 \mathrm{~km} \mathrm{~s}^{-1}$ (observed) for the red side - this is roughly the NE side of the disk (top panel), hence, the blue side is the SW side of the disk (bottom panel). The black line is for the central position (the continuum peak), while the yellow, orange, and red (green, cyan, blue) lines are for beam offsets $\theta_{B} / 2, \theta_{B}$, and $1.5 \theta_{B}$ along the major axis for the red (blue) sides of the disk.

The model makes a number of generic predictions that can be usefully compared with the observed spectra along the disk major axis:

1. The disk center is where we see a symmetric, double-peaked line profile.

2. The blue/red line peak brightnesses occur away from the center position.

3. The blue/red line peak brightnesses and the extent 

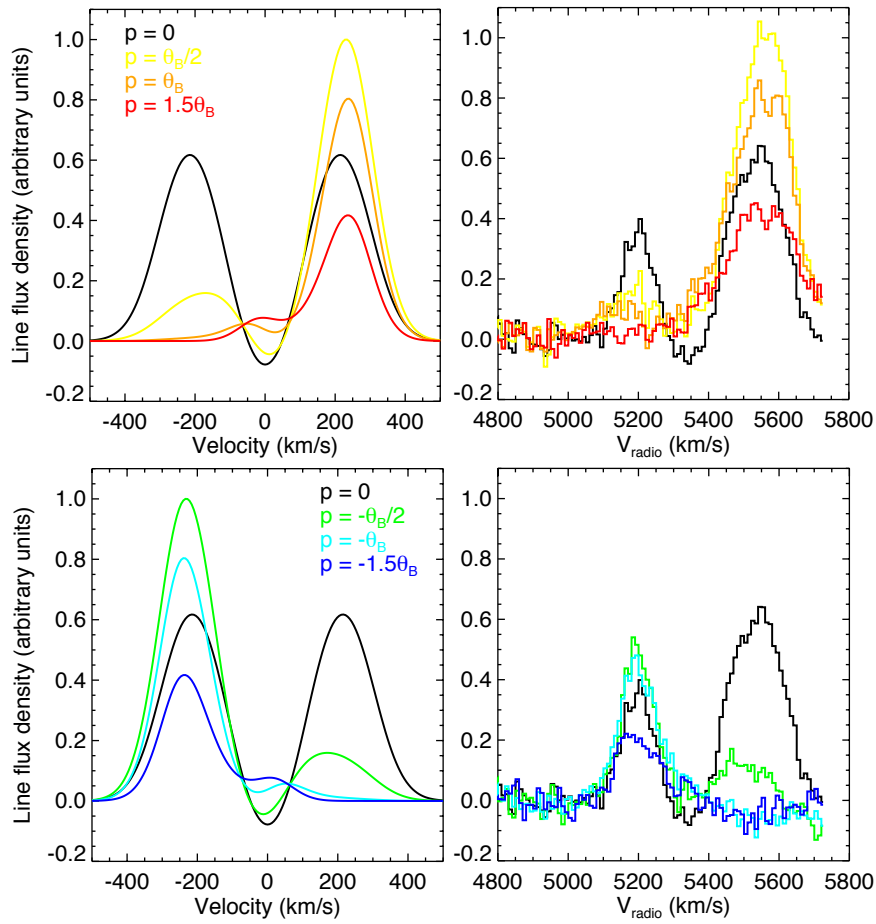

Figure 7. Comparison between the velocity profiles generated from an axisymmetric disk model and observations for the NE (top panels) and SW (bottom panels) sides of the eastern nucleus disk along the major axis of rotation. Black, yellow, orange and red (green, cyan, and blue) are for offsets of $0, \theta_{B} / 2, \theta_{B}$, and $1.5 \theta_{B}$ away from the continuum center along the major axis approximately towards the NE (SW) side of the disk.

of the disk should be symmetric about the disk center.

4. Emission in the blue wing is still present at a low level as one moves redward of the disk center, and vice versa.

5. Producing line profiles that are as broad and approximately Gaussian as observed requires a lineof-sight velocity dispersion that is comparable to the rotation velocity. See the appendix for a discussion of what constraints the line profiles place on $\sigma / V_{\text {rot }}$.

Prediction (1) immediately implies that the disk center is at the continuum peak, not at the peak of the line emission, i.e., even though we find a 100 pc shift between the line peak and the continuum peak, the center of the disk is located at the continuum peak. This is what we would expect, as the dust emission is unaffected by the disk kinematics. It also implies that the central velocity of the deep absorption dip is the systemic velocity.

Prediction (2), which is the inevitable result for a rotating disk with a source function gradient when the optical depth is not extremely high along all lines of sight, also agrees with the observations, but more so on the red (NE) side of the disk than the blue (SW) side. The disagreement becomes much more pronounced with the third prediction: the blue line peak maximum is considerably weaker than the red peak, and the disk extent on the blue side is noticeably less than that on the red side. Figure 7 shows that, while the ordering of peak flux density with position is basically correct, the observed line peak values are too small by about a factor of two compared to the model. In addition, the observed line widths of the blueshifted lines are systematically smaller than those of the redshifted lines, also by about a factor of two.
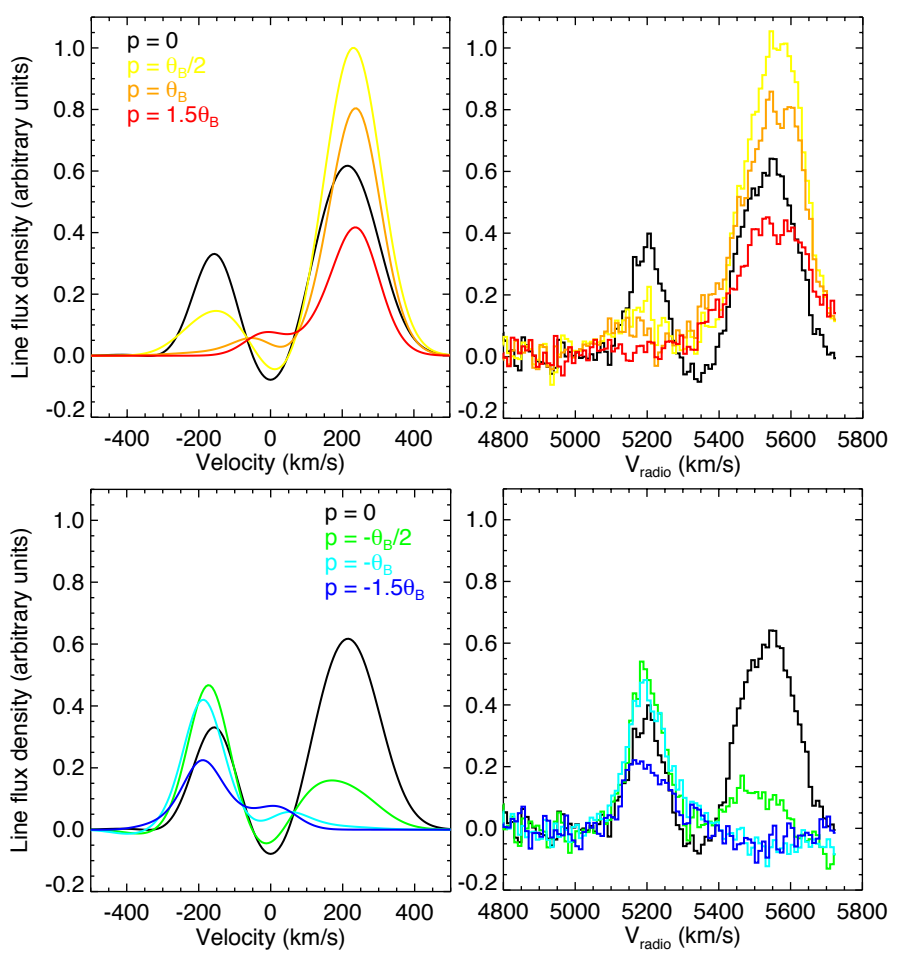

Figure 8. Comparison of the eastern nucleus kinematics between the axisymmetric disk plus foreground absorption model and observations for the SW and NE sides of the nuclear disk along the major axis of rotation. Color coding as in Figure 7

The discrepancy with the axisymmetric model could be the result simply of asymmetry in the disk, in excitation, optical depth (e.g., the disk column density), or local kinematics - the disk dynamical timescale (radius of $100-200 \mathrm{pc}$, rotation velocity $\sim 130 \mathrm{~km} \mathrm{~s}^{-1}$ ) is a few million years. However, both the eastern and western nuclei show asymmetry in the same sense: although the $\mathrm{CO}$ emission is more extended than the continuum, the line emission in both nuclei is systematically weaker on the southern side of the disks (which is the blue side for the eastern nucleus), compared to the continuum extent (see Figure 2(a,e,f)).

There are two candidate mechanisms for producing such asymmetries in the line emission, absorption and outflow. We consider these in turn in the following subsections.

\subsection{Rotating disk models with foreground absorption}

The commonality of the $\mathrm{CO}$ line asymmetry in both nuclei suggests that something external to the nuclei is the cause. We therefore model it as additional, foreground absorption of the disk emission. This model assumes a Gaussian absorption profile in velocity characterized by the line center velocity $V_{a b s}$, velocity dispersion $\sigma_{a b s}$, and line center optical depth $\tau_{a b s}$, which are all allowed to vary with position. In practice, $\sigma_{a b s}=70$ $\mathrm{km} \mathrm{s}^{-1}$ provided a match in all cases. Because the 
line/continuum level is very important in determining the optical depth, we tweaked the model continuum levels slightly to precisely match the observations.

Consider first the blue side of the disk, where we see large discrepancies between the original unabsorbed model and the data for blue velocities. The absorbed model emission profiles (after continuum subtraction) are shown in Figure 8 , Given the simplicity of the model, the agreement is very good. Interestingly, there are signs of gradients as a function of position in both the absorption velocity and (clearly) in optical depth: the former shifts further away from zero velocity by about $40 \mathrm{~km} \mathrm{~s}^{-1}$, while the latter increases by nearly a factor of $3\left(\tau_{0}=0.5\right.$, $\left.\tau_{-\theta_{B} / 2}=0.9, \tau_{-\theta_{B}}=1.3, \tau_{-3 \theta_{B} / 2}=1.5\right)$. On the red side of the disk, only very weak absorption is allowed at $p=\theta_{B} / 2$, with $\tau$ no more than $\sim 0.1$, and basically none at larger offsets. (Indeed, at $p=\theta_{B}$, there is clearly emission at these velocities that is not present in the model.) Hence in this model there is little to no absorption on the red (NE) side, but the absorption optical depth is significant at the continuum peak and it increases rapidly to the SW with distance from the center.

Where might this absorbing gas lie? One possibility is that both absorption features, i.e., the absorption that reduces the blue emission peak and the deep redshifted absorption (seen above $5505 \mathrm{~km} / \mathrm{s}$ ) are produced in one more-or-less contiguous feature. This would suggest that there is a very broad ( $\gtrsim 650 \mathrm{~km} \mathrm{~s}^{-1}$ in velocity space) complex of absorbing gas, the bulk of which is flowing outwards (blueshifted with respect to the systemic velocity), assuming that optical depth (which is probably larger for the blueshifted absorption than for the redshifted) traces column density. The lower mass tail of absorbing gas extending to redshifted velocities may represent dynamical spraying of the absorbing gas over a wide range of velocities, e.g., by tidal or ram-pressure stripping.

To explain the common CO asymmetry and redshifted absorption of the nuclei, this gas must be at least as spatially extended as the nuclear separation ( $\sim 400 \mathrm{pc})$. Given the complicated dynamics of the merging galaxies, the existence of such a spatial and kinematic feature is certainly plausible. Furthermore, the spatial location of the blue absorber would be coincident with the location of the redshifted absorption (Figures 2(f)), suggesting a physical connection between the two.

Alternatively, the blue-shifted absorbing gas causing the apparent line/continuum peak shift and the blue/red disk asymmetry could also be explained by an outer, kiloparsec-scale molecular disk, inferred by Sakamoto et al. (2009) and Scoville et al. (1997) from low- $J$ CO interferometric observations. If this outer disk is suitably tilted with respect to the two embedded nuclear disks, then the blue sides of the nuclear disks will appear fainter than the red sides as a result of absorption. The magnitude of the required velocity dispersion $\left(\sim 70 \mathrm{~km} \mathrm{~s}^{-1}\right)$ is certainly reasonable for such a disk, especially as it has quite likely not settled into dynamical equilibrium.

\subsection{Rotating disk models with outflow}

Sakamoto et al. (2009) suggested that outflows with velocity $V_{\text {out }} \sim 100 \mathrm{~km} \mathrm{~s}^{-1}$ are present in the nuclei of
Arp 220, based on their reported detection of P Cygni profiles in the $\mathrm{HCO}^{+}(4-3)$ and (3-2) lines towards both nuclei and in the CO $J=3-2$ line for the eastern nucleus, in SMA data with $0.3^{\prime \prime}$ resolution. Rotation is also clearly present in their data, and their preferred model included both rotation and outflow.

With their more limited data set, they concluded that the absorption dip seen in the $\mathrm{CO}$ and $\mathrm{HCO}^{+}$lines from the eastern nucleus is blueshifted by $\sim 100 \mathrm{~km} \mathrm{~s}^{-1}$ with respect to the systemic velocity, which they estimated to be $V_{\text {sys }}=5415 \pm 15 \mathrm{~km} \mathrm{~s}^{-1}$; a blueshift of the absorption must be present if an outflow is responsible for the line profile asymmetries. In Figure 9 we overplot their line profiles on ours, for both the eastern (left panel) and western (right panel) nucleus. For the eastern nucleus it is clear that the velocities of the emission peaks and of the absorption dip for all three transitions are consistent, although the degree of asymmetry differs. We defer discussion of the velocity shift of the absorption relative to the systemic velocity to $\S 4.4$.

Since rotation is undoubtedly present, and the parameter space for rotation plus outflow models is large, we adopted the same axisymmetric rotating disk model as in $\S 4.1$. As models in which there is a gradient in outflow velocity, rather than a constant velocity, provided better matches, we assumed a linear increase in outflow velocity. (Models with exponential instead of linear rises differ insignificantly). The model outflow is symmetric about the line of sight and has a half-opening angle $\theta_{\text {out }}$. In practice, we found that models with $\theta_{\text {out }} \geq 30^{\circ}$ were indistinguishable from models with larger half-angles, and provided better fits than narrower outflows.

Reasonable approximations to the observed profiles are produced by models with $V_{\text {out }} \simeq 100 \mathrm{~km} \mathrm{~s}^{-1}$. The resulting profiles are compared with the observed profiles in Figure 10, In detail the rotation plus outflow models do not match the data as well as the rotation plus absorption models shown in Figure 8, especially on the blue side of the disk, where they tend to produce too much emission in the red wing of the lines. However, this conclusion should not be considered in any way definitive, since the amount of unexplored parameter space is very large and we did not vary the underlying axisymmetric rotating disk model, only the outflow parameters. Hence it is only fair to conclude that the rotation plus outflow models for the eastern nucleus also provide an acceptable explanation for the observed line profiles.

\subsection{The Observed and Actual Disk Velocity Curves}

We extract the observed rotation curve for the eastern nucleus from the moment-1 map shown in Figure 2(b) by taking a cut along the major axis. This is shown in Figure 11. The curve has been color-coded as red- and blue-shifted with respect to the systemic velocity, which has here been defined to be the mean velocity at the position of the continuum peak, which is $5450 \mathrm{~km} \mathrm{~s}^{-1}$. To be specific, what is plotted here - the quantity calculated by moment-1 - is the intensity-weighted mean velocity (IWMV) for each pixel along the major axis. This weighting has an important influence on the relation between the observed and actual rotation curves. Also, since the uncertainty in the mean velocity curve is completely dominated by the uncertainty in the position angle of the major axis, we have overplotted IWMV 

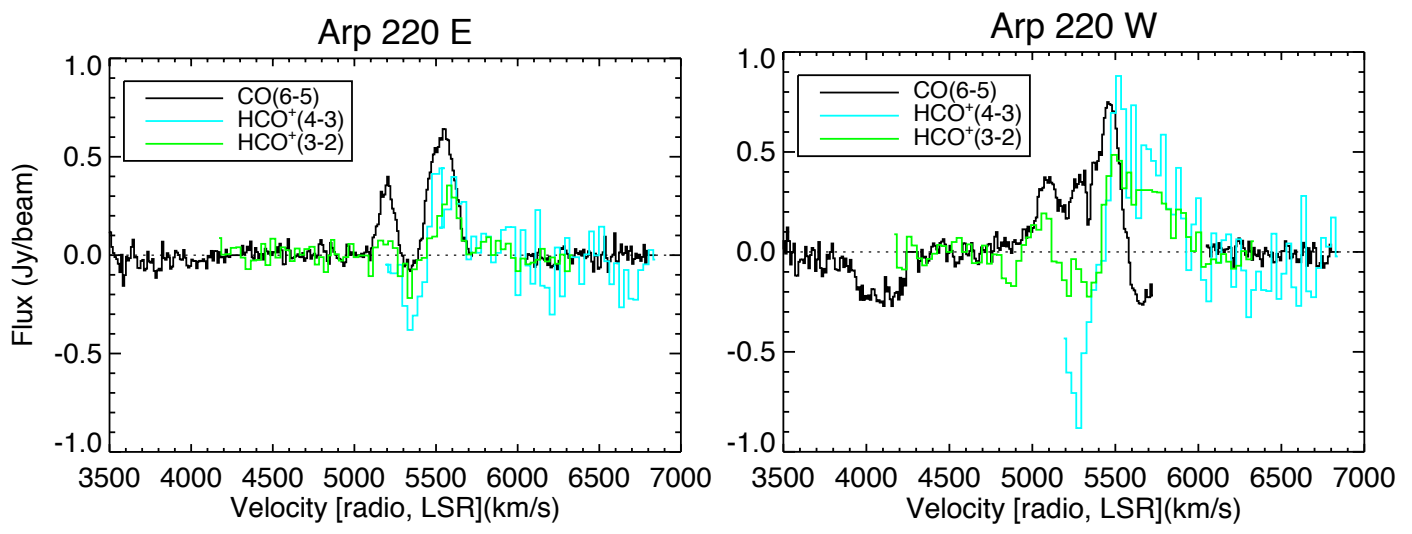

Figure 9. Comparison of $\mathrm{CO} J=6-5$ line profile (this work) with the $\mathrm{HCO}^{+}(4-3) /(3-2)$ lines profiles from Sakamoto et al. (2009).
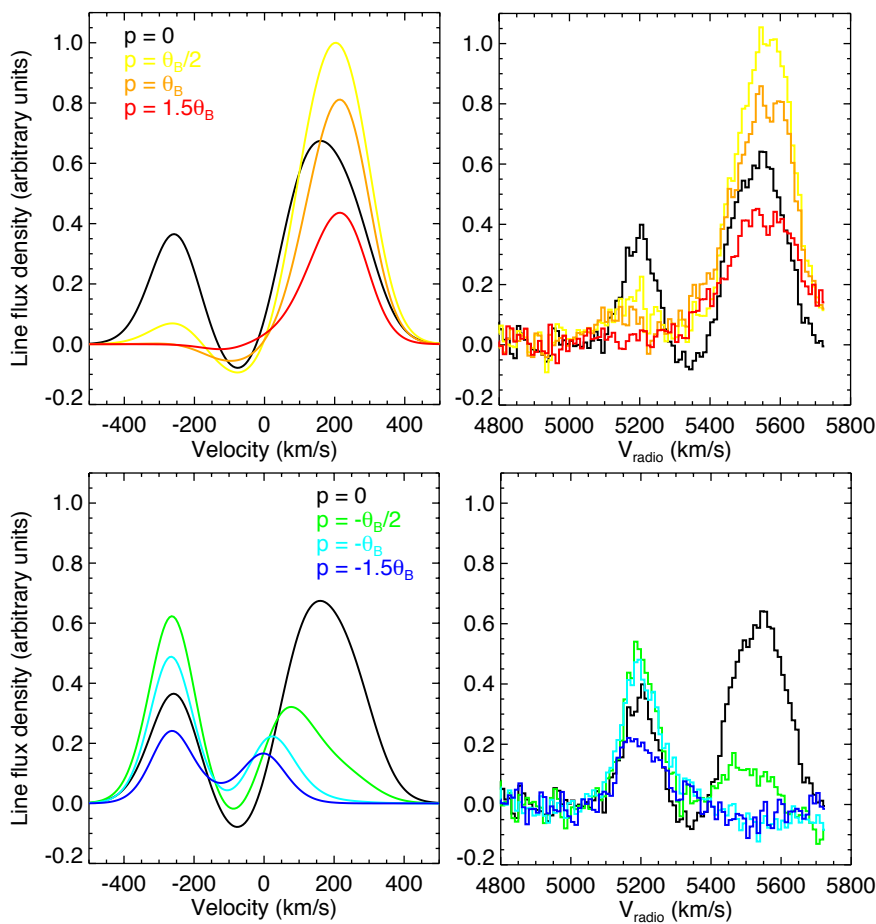

Figure 10. Comparison of the eastern nucleus kinematics between the axisymmetric disk plus outflow model and observations for the SW and NE sides of the nuclear disk along the major axis of rotation. Color coding as in Figure 7

curves (with similar color-coding) for position angles between $28^{\circ}$ and $36^{\circ}$, which covers the range of uncertainty in the major axis position angle based on the continuum emission (which is unaffected by absorption) presented in Paper I. The variation between them is insignificant.

As mentioned in $\S 4.1$, the axisymmetric rotating disk model predicts that the continuum peak is the true center of the nucleus, and the central velocity of the selfabsorption dip is the systemic velocity. This velocity is $5350 \mathrm{~km} \mathrm{~s}^{-1}$ (e.g., Figure 2(a)). Hence there is a difference of approximately $100 \mathrm{~km} \mathrm{~s}^{-1}$ from the IWMV at the continuum peak. In addition, the observed rotation curve is noticeably asymmetric about the continuum peak IWMV. The red side is much shallower than the blue side: at $100 \mathrm{pc}$ distance from the continuum peak, the velocity has risen by less than $100 \mathrm{~km} \mathrm{~s}^{-1}$ with respect to systemic velocity, whereas on the blue side it is

over $200 \mathrm{~km} \mathrm{~s}^{-1}$.

However, the asymmetry of the observed line profiles, and in particular the difference in profiles between the red and blue sides of the disk, produces a systematic offset between the IWMV and the true velocity centroids of the emission. In the presence of such line asymmetries (whatever their origin), IWMV curves can be very misleading about the underlying kinematics. As we now show, the intensity weighting of an asymmetric line profile can result in an IWMV curve which has both an offset from the true systemic velocity and an asymmetry in the derived rotation curve about the disk center, even though the underlying kinematic model is purely axisymmetric.

Figure 12 (top) shows the IWMV curve produced by the axisymmetric model of $\S 4.1$, in the absence of absorption (solid line). This is color-coded as in Figure 11. and is of course symmetric about the disk center, since the line profiles, although asymmetric except for $p=0$, are mirror images of one another on the red and blue sides of the disk. We define the apparent systemic velocity to be the IWMV at zero offset from the continuum peak (identical to the disk center), just as in Figure 11] for the unabsorbed model this is of course equal to the true systemic velocity of $0 \mathrm{~km} / \mathrm{s}$. 7 The dashed line shows the same thing, but now for the absorbed model of $\S 4.2$. On the red side of the disk, the absorption rapidly goes to zero with increasing distance from the disk center, so the red with-absorption curve converges with the no-absorption curve. Since the effect of absorption is to reduce the blue wing of emission, the absorbed IWMV curve shifts closer to zero on the blue side, and away from zero on the red side, with the result that the absorbed IWMV curve shifts upward with respect to the unabsorbed IWMV curve.

However, the inferred systemic velocity is no longer zero; it has been offset to the red, with $V_{\text {sys }} \simeq 125$ $\mathrm{km} \mathrm{s}^{-1}$. (Recall that for the observed disk, the velocity offset between the absorption dip central velocity, which should be the systemic velocity, and the IWMV at the continuum peak is $\Delta V \simeq 100 \mathrm{~km} \mathrm{~s}^{-1}$ to the red.) In the lower panel we have subtracted off this apparent

\footnotetext{
${ }^{7}$ It is worth noting the weak resemblance between this "rotation curve" and the true disk rotation curve shown in Figure 7 This is a consequence of several systematic biases, especially the $\cos \theta$ weighting of the line-of-sight velocity component, the relatively poor spatial resolution of the disk, the optical thickness of the lines, and the intensity-weighting used to derive the mean velocity.
} 
$V_{\text {sys }}$ from the absorbed model curve. This curve now exhibits all of the features shown by the observed IWMV curve: a steeper rise (and greater range) on the blue side, and a shallower red IWMV curve. Hence the observed IWMV curve is completely consistent with an underlying axisymmetric disk model, once the effects of the line profile asymmetries are taken into account.

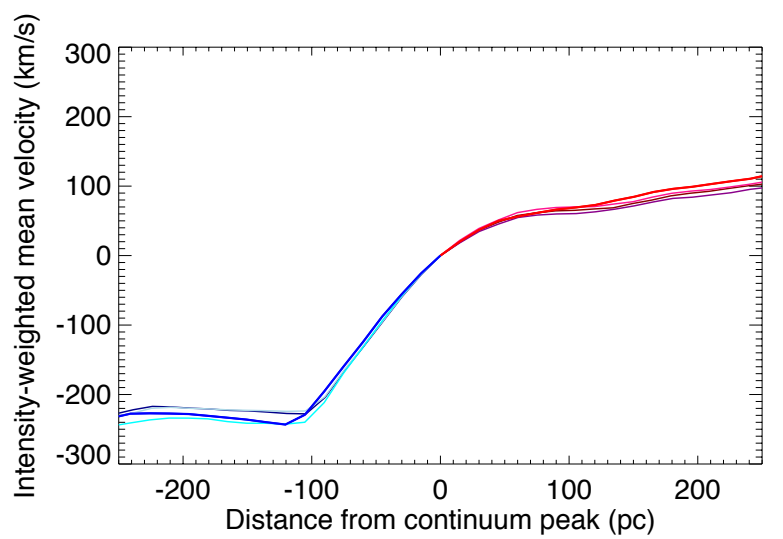

Figure 11. Observed IWMV rotation curve along the major axis of the eastern nucleus. The apparent systemic velocity is defined to be the IMWV at the continuum peak, $5450 \mathrm{~km} \mathrm{~s}^{-1}$. We have subtracted this quantity so that the plotted curves show rotation about the apparent systemic velocity, with the approaching and receding sides of the disk color-coded appropriately. Distances are positive in the NE direction (the red side of the disk). Rotation curves for position angles between $28^{\circ}$ and $36^{\circ}$ are overplotted to show that the variations between them are extremely small.

In Figure 13 we show the same pair of IWMV curves as in Figure 12 for the outflow model of $\S 4.3$. Although the overall effect of the outflow is similar to that for absorption, the apparent red side/blue side IWMV curves are noticeably less asymmetric than for the absorbed model, or as seen in the data. This is because the outflow inevitably shifts line emission blueward from where it would occur in the absence of the outflow: as seen in Figure 10, the absorption dip has shifted by about $75 \mathrm{~km} \mathrm{~s}^{-1}$ to the blue from the true systemic velocity, and so part of the red wing of the line profile has also shifted to the blue, something which does not happen in the absorption model. In consequence, the IWMV at the continuum peak has shifted to the red by only about half as much as in the absorption model (about $68 \mathrm{~km} \mathrm{~s}^{-1} \mathrm{vs}$ $125 \mathrm{~km} \mathrm{~s}^{-1}$ ), and the two sides of the IWMV curves are more symmetric and less similar to the observed IMWV.

While not a definitive argument, this does suggest that outflow models are less capable of matching the observed kinematic data than the absorption models.

\subsection{Rotation plus foreground absorption or rotation plus outflow?}

The line profiles and kinematics of the eastern disk are fit about equally well by a rotating, axisymmetric disk model combined with either foreground absorption on the blue side of the disk or with an outflow. On balance, however, we favor absorption as the explanation for the observed asymmetries in the line profiles and the disk extent with respect to the continuum peak:

1. There is unequivocally foreground absorption present at redshifted (with respect to systemic) ve-

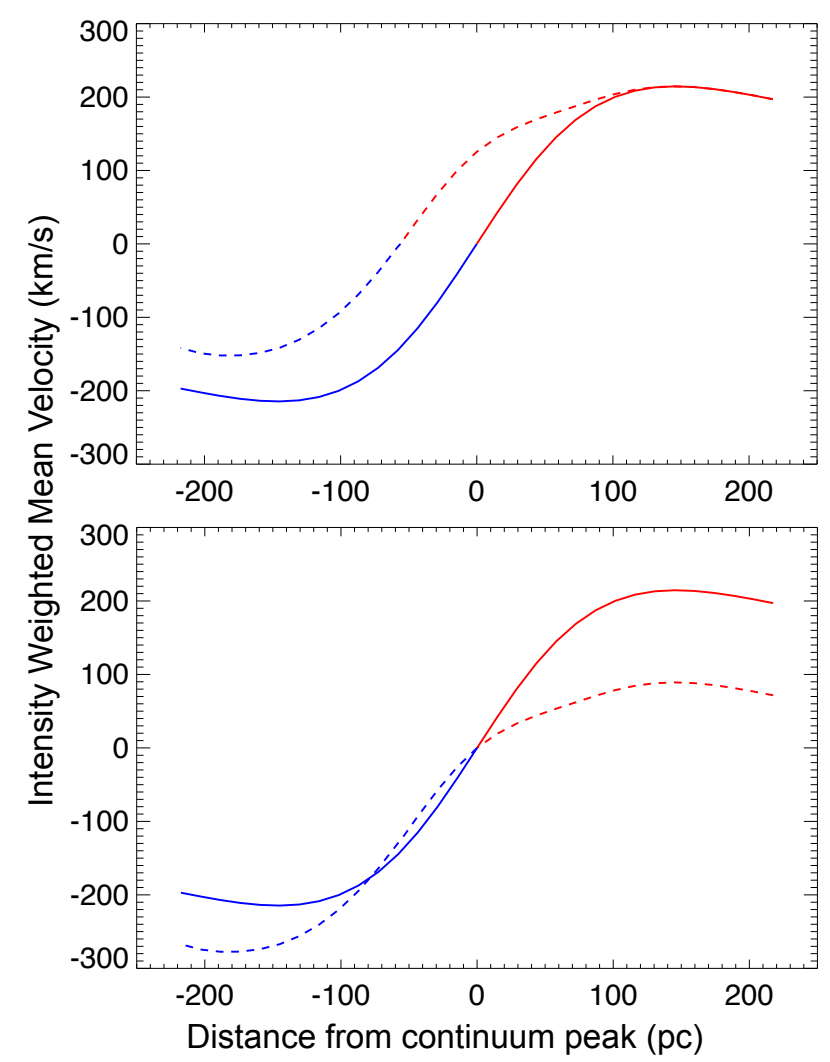

Figure 12. Rotation plus foreground absorption: Model IWMV curves, color-coded as in Figure 11 Top: Axisymmetric rotating model IWMV curve (solid line); with absorption (dashed line). The apparent systemic velocity is defined (as in Figure 11) as the IMWV at zero offset from the disk center. With no absorption this is at the true systemic velocity, $0 \mathrm{~km} / \mathrm{s}$. The effect of absorption is to shift this velocity to the red by $\simeq 125 \mathrm{~km} \mathrm{~s}^{-1}$. Bottom: As the top panel, except that now the apparent systemic velocity has been subtracted from the dashed curve. Bottom: As the top panel, except that now the apparent systemic velocity (the IWMV at zero offset from the disk center) has been subtracted from the dashed curve.

locities, over at least a few hundred $\mathrm{km} \mathrm{s}^{-1}$, and it occurs spatially precisely where the blue shifted absorption is expected to lie. Given the complicated dynamics and energetic nuclei present in Arp 220, the presence of additional absorption at blueshifted velocities (representing outflowing rather than infalling gas) would not be unexpected.

2. The line asymmetries produced by an outflow depend rather sensitively on the magnitude of the projected velocity compared to other velocities in the system, such as rotation. However, even though the two nuclear disks have very different apparent orientations, the asymmetries between the integrated line flux and the continuum emission for the two disks is very similar, as seen on the sky. This is natural in the absorption picture (the absorber only needs to be at least as large as the nuclear separation, and simply absorbs background radiation over its velocity extent) but rather contrived in the outflow model, where despite the different orientations of the nuclei, the projected rotation and outflow velocities conspire in such a way as to cause the line emission on the south sides of the disk to fade out with respect to the continuum in 


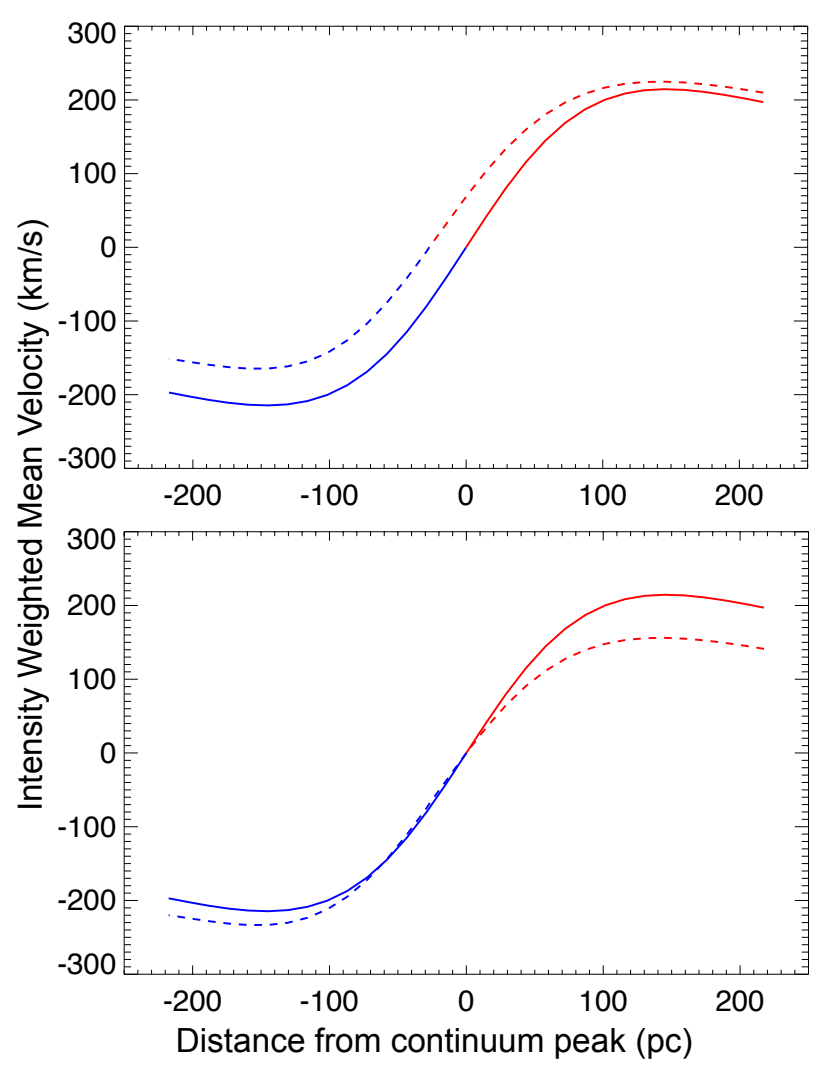

Figure 13. Rotation plus outflow: Model IWMV curves, colorcoded as in Figure 11 Top: Axisymmetric rotating model IWMV curve (solid line); with outflow (dashed line). Bottom: As the top panel, except that now the apparent systemic velocity (the IWMV at zero offset from the disk center) has been subtracted from the dashed curve.

very similar fashion. (Recall that although we have not modeled the western nuclear disk, similar line profile asymmetries are seen there, e.g., Figure 9])

3. Despite the different orientations of the nuclei, the gas velocities on the south sides of the two nuclei (where the absorption occurs) are very similar (Figure 2(b)): from the eastern to the western nucleus on the blue side, all of the emission falls within the same range (covering $\sim 100 \mathrm{~km} \mathrm{~s}^{-1}$ ) so the absorbing gas can occupy the same range of velocities over its whole extent, with no need to introduce a velocity gradient across the absorber.

4. The IWMV curve produced by the rotation plus absorption model resembles the observed curve much more closely than the rotation plus outflow model, in considerable part because in the outflow model some of the red side emission must be shifted blueward.

The rotation plus absorption model still requires that there is outflowing, blueshifted gas in the Arp 220 system. However, this gas is not dynamically responsible for the line asymmetries. Whichever model is correct, however, the mass of gas involved in the outflow is very substantial. In the rotation plus outflow model, a substantial fraction of the total mass of the disk (for which we estimate a warm gas mass $M_{\mathrm{H}_{2}} \simeq 1.3 \times 10^{8} M_{\odot}$, see Table 1) must be participating in the outflow in order to produce the observed line profile asymmetries, the exact amount depending on the opening angle of the outflow. This also implies that the lifetime of the disk is only $t_{\text {out }} \sim R / V_{\text {out }} \sim 10^{6} \mathrm{yr}$.

For the rotation plus absorber model, the mass is more uncertain. We can write the line center optical depth in a Doppler-broadened line as

$$
\tau_{0}=\frac{c^{3}}{8 \pi^{3 / 2} \nu_{i}^{3}} A_{j i} \frac{g_{j}}{g_{i}} \frac{N_{i}}{\Delta V}\left(1-e^{-h \nu_{i} / k T_{e x}}\right)
$$

where $\nu_{i}$ is the line frequency, $A_{j i}$ is the Einstein $A$ coefficient, the $g_{i}$ and $g_{j}$ are the statistical weights of the levels, $N_{i}$ is the column density in the lower state of the transition, and $\Delta V$ is the Doppler width. For the $\mathrm{CO}$ $J=6-5$ line, $A_{j i}=2.126 \times 10^{-5} \mathrm{~s}^{-1}$ and $\nu=691.473$ GHz. For the blue-side absorber, $\Delta V=\sqrt{2} \times 70 \mathrm{~km} \mathrm{~s}^{-1}$ and $\tau_{0} \sim 1$. The $\mathrm{H}_{2}$ column density corresponding to equation 10 is then

$N_{\mathrm{H}_{2}, a b s}=2.2 \times 10^{21} \frac{Q_{R}}{\left(x_{\mathrm{CO}} / 10^{-4}\right)}\left(1-e^{-33.2 / T_{e x}}\right)^{-1} \mathrm{~cm}^{-2}$

where $x_{\mathrm{CO}}=10^{-4}$ is the $\mathrm{CO}$ abundance with respect to $\mathrm{H}_{2}$ and $Q_{R}=\sum g_{i} e^{-h \nu_{i} / k T_{e x}}$ is the rotational partition function for $\mathrm{CO}$.

Spatially, the absorber must extend at least $\sim 400 \mathrm{pc}$ (the separation of the nuclei) by $220 \mathrm{pc}$ (the extent of the observed absorption on the SW side of the eastern nuclear disk), giving a minimum area $A_{a b s} \approx 8.8 \times 10^{4}$ $\mathrm{pc}^{2}$. The minimum mass of the absorber is then

$$
\begin{aligned}
M_{a b s} & >A_{a b s} N_{\mathrm{H}_{2}} \mu m_{\mathrm{H}_{2}} \\
& \approx 4.2 \times 10^{6} \frac{Q_{R}}{\left(x_{\mathrm{CO}} / 10^{-4}\right)}\left(1-e^{-33.2 / T_{e x}}\right)^{-1} M(12)
\end{aligned}
$$

where $\mu$ is the mean molecular weight per $\mathrm{H}_{2}$ molecule.

The partition function $Q_{R}$ is almost certainly $>10$, and plausibly as large as 100 , while the correction for stimulated emission is likely $\sim 1$ to a few, giving

$$
M_{a b s} \sim \text { few } \times\left(10^{7}-10^{8}\right) M_{\odot} .
$$

This is again a very substantial amount of mass, comparable to the total mass of warm molecular gas in Arp 220 (Note: The warm gas mass in Arp 220 is $\sim 10 \%$ of the total gas mass). The velocity characterizing the absorber is much larger than in the outflow model, reaching blueshifted velocities of nearly $300 \mathrm{~km} \mathrm{~s}^{-1}$ with respect to systemic, as this gas must absorb the blue wings of the emission lines.

An interesting question is whether we should expect to see the blue absorber emission away from the nuclear disks. Exploratory non-LTE modeling using RADEX (van der Tak et al. 2007) of plausible absorber parameters (defined as those which produce $Q_{R}$ not much larger than 10 , so as to minimize the mass of the absorber) suggests that the ratio of warm gas to absorber peak antenna temperatures in the $J=6-5$ line is close to a factor of 10 . Furthermore the velocity extent of the absorber is almost certainly substantially smaller than that of the warm gas emission, further increasing the ratio in integrated intensity. Comparison with Figure 2(a) suggests that it is unlikely that the absorber emission is detectable on top of the general nuclear disk emission. 
There is clearly extended emission present in the channel maps between $5105 \mathrm{~km} \mathrm{~s}^{-1}$ and $5225 \mathrm{~km} \mathrm{~s}^{-1}$, where we would expect the blue absorber emission to exist, but its impossible to conclude if this is from the absorbing gas rather than the general disk emission.

ALMA observations of other CO transitions (e.g., $J=$ $4-3,7-6)$ will be crucial in constraining the partition function, $Q_{R}$, and excitation temperature, $T_{e x}$, by providing estimates of the variation of line optical depth with $J$, and thus reducing the uncertainty on the mass estimate of the absorber. For example, the same absorber models described above predict that the absorber is considerably brighter in the $J=3-2$ line, both absolutely and relative to the warm gas (cf. end of $\S 3$ and Figure 4).

\section{DYNAMICAL MASS AND DISK STABILITY}

The numbers derived from the kinematic modeling in $\S 4$ can be used to estimate the enclosed mass in the eastern nucleus. Adding the asymptotic rotation velocity $\left(135 \mathrm{~km} \mathrm{~s}^{-1}\right)$ and velocity dispersion $\left(85 \mathrm{~km} \mathrm{~s}^{-1}\right)$ in quadrature, we derive a dynamical mass within $r=220$ pc of $M_{\text {tot }} \sim 1.6-2.9 \times 10^{9} M_{\odot}$, where the larger number is obtained by correcting the rotation velocity (but not the velocity dispersion) for an inclination of $50^{\circ}$, obtained by Barcos-Muñoz et al. (2014) from a two-dimensional nonlinear least-squares fit of a thin, tilted, exponential disk to their $33 \mathrm{GHz}$ radio continuum image with $0.08^{\prime \prime}$ $\mathrm{x} 0.06^{\prime \prime}$ resolution. Given the uncertainties, this enclosed mass is in agreement with the total molecular gas mass of $1.1 \times 10^{9}$ derived in Paper I from the continuum emission (adopting a gas-to-dust mass ratio of 100). This implies that the dynamical mass within the radius of $220 \mathrm{pc}$ is largely dominated by the molecular gas, consistent with previous studies of Arp 220.

An interesting question is whether the nuclear disks are liable to be gravitationally unstable to fragmentation and the formation of stars given the large inferred mass surface densities (see Paper I) of about $5.4 \times 10^{4} \mathrm{M}_{\odot} / \mathrm{pc}^{2}$ and $1.4 \times 10^{5} \mathrm{M}_{\odot} / \mathrm{pc}^{2}$ associated with the disks of the eastern and western nuclei, respectively. We investigate this using our model for the eastern nucleus and the Toomre $Q$-parameter for a gaseous disk (Toomre 1964), in the form

$$
Q \equiv \frac{v_{s} \kappa}{\pi G \Sigma}>1
$$

for stability, where $v_{s}$ is the sound speed in the gas, $\kappa$ is the epicyclic frequency, $G$ is the gravitational constant, and $\Sigma$ is the gas surface density 8

To evaluate $\Sigma$, we use our dynamical mass estimate $M_{d} \sim 2 \times 10^{9} M_{\odot}$ and an effective disk area $A=\pi \theta_{E}^{2}$, where $\theta_{E}=276 \mathrm{pc}$ is the deconvolved FWHM for the eastern nucleus. This gives $\Sigma \approx 8400 M_{\odot} \mathrm{pc}^{-2}$. Note that this is an average surface density (and thus lower) compared to the value reported in paper I, which was effectively the peak surface density calculated on a smaller spatial scale (radius $<50 \mathrm{pc}$ ). We also assume a flat rotation curve with $V_{c}=135 \mathrm{~km} \mathrm{~s}^{-1}$, so that $\kappa=\sqrt{2} \Omega=\sqrt{2} V / R$, which we evaluate at $R=\theta_{E} / 2$ as

\footnotetext{
${ }^{8}$ Strictly speaking, this equation applies to a thin disk, which is not the case here, since the ratio of the velocity dispersion to the rotation velocity $\sigma / V_{c} \approx 0.5-1$. The effect of this will be to make the disk more stable than implied by equation (14).
}

a typical value. Finally, we use the inferred velocity dispersion $\sigma=85 \mathrm{~km} \mathrm{~s}^{-1}$ as an effective sound speed. With these parameters, we find $Q \sim 1.5$; this number should be regarded as uncertain by at least a factor of two (furthermore, $\Sigma, V$, and $\sigma$ are all functions of radius). However, the estimated Toomre Q-parameter for the gas disk of the eastern nucleus is of order unity, suggesting that the disk is marginally stabilized against gravitational fragmentation and collapse by the large velocity dispersion. This large velocity dispersion requires energy input to sustain it unless we are catching Arp 220 in a very short-lived phase. The amount of random (non-rotational) kinetic energy in the ISM can be written

$$
E_{k i n} \sim \frac{1}{2} M \sigma^{2} \sim 10^{56}\left(\frac{M}{10^{9} M_{\odot}}\right) \sigma_{100}^{2} \text { erg }
$$

where $M$ is the mass of molecular gas and $\sigma_{100}$ is the velocity dispersion in units of $100 \mathrm{~km} \mathrm{~s}^{-1}$. Dimensionally, the energy dissipation rate is (Malonev 1999)

$$
\begin{aligned}
\dot{E}_{k i n} & \sim \eta \frac{M}{R} \sigma^{3} \\
& \sim 6 \times 10^{42} \eta\left(\frac{M}{10^{9} M_{\odot}}\right) R_{100}^{-1} \sigma_{100}^{3} \mathrm{erg} \mathrm{s}^{-1}
\end{aligned}
$$

where $R=100 R_{100} \mathrm{pc}$ is a characteristic radius and the coefficient $\eta \lesssim 1$ (Mac Low 1999). The ratio of these two equations gives a dissipation time

$$
t_{d i s s}=\frac{E_{k i n}}{\dot{E}_{k i n}} \sim 5 \times 10^{5} \frac{R_{100}}{\eta \sigma_{100}} \mathrm{yr} .
$$

(A very similar estimate is obtained simply from assuming that the areal filling factor is roughly unity and that collisions are strongly dissipative.) For the eastern nucleus of Arp 220, $R_{100} \sim \sigma_{100} \sim 1$, which gives the dissipation time of only $t_{\text {diss }} \sim 10^{6} \mathrm{yr}$. This short timescale implies that there must be an energy input sustaining the large velocity dispersion.

\section{WARM MOLECULAR GAS MASS AND LUMINOSITY}

We determined the mass of the warm molecular gas (listed in Table 1) in the individual nuclei using the information from our Herschel SPIRE-FTS observations of Arp 220 (Rangwala et al. 2011). We derived the ratio of the total warm molecular gas mass to the total $\mathrm{CO}$ $\mathrm{J}=6-5$ line luminosity in Arp 220; the former is derived from non-LTE modeling of the full CO SLED from $J=1-0$ to $J=13-12$ and the latter is measured directly from observations. This ratio $M\left(\mathrm{H}_{2}\right) / L_{\mathrm{CO}}(6-5)$ is about $21 \mathrm{M}_{\odot} / \mathrm{L}_{\odot}$. Using this ratio and the individual integrated CO $J=6-5$ ALMA fluxes for the two nuclei we estimate the warm molecular gas mass to be $1.4 \times 10^{8} \mathrm{M}_{\odot}$ and $1.6 \times 10^{8} \mathrm{M}_{\odot}$, in the eastern and western nucleus, respectively 9 These are roughly $1 / 10$ th of the total molecular gas masses measured from dust continuum emission in Paper I and are consistent with the warm molecular gas mass fraction found in nearby galaxies (Kamenetzky et al. 2014).

9 We note that the beam size of Herschel is large compared to the angular extent of Arp 220 and therefore this ratio is an average quantity over the whole system and is likely to vary spatially. Hence, our mass estimate for the warm molecular gas for the individual nuclei should be used with caution. 
We list the observed $L_{\mathrm{COJ}=6-5} / L_{\mathrm{FIR}}$ ratio in the two nuclei in Table 1 using the $L_{\text {FIR }}$ value calculated in Paper I. This ratio differs by almost an order of magnitude between the two nuclei - a direct result of their $L_{\text {FIR }}$ values differing by an order of magnitude. The ratio in the eastern nucleus of $\sim 6.7 \times 10^{-5}$ is much higher than the western nucleus; the value for the latter is within a factor of two of the average value of $\sim 10^{-5}$ found in nearby galaxies (Kamenetzky et al. 2014). Despite the disparity in the ratio of $\mathrm{CO} J=6-5$ luminosity to bolometric luminosity, the line-to-continuum ratios at $691 \mathrm{GHz}$ (listed in Table 1) are the same for the two nuclei, implying that the eastern nucleus contains an additional power source which is depositing energy into the gas without affecting the dust continuum emission. Such an energy source is consistent with mechanical energy from shocks. A similar scenario is inferred in another nearby IR-luminous galaxy, NGC 6240, in which the CO is also unusually luminous relative to the bolometric luminosity, with $L_{\mathrm{COJ}=6-5} / L_{\mathrm{FIR}} \sim 7.6 \times 10^{-5}$ (Meijerink et al. 2013). It is intriguing that even though there is evidence of large-scale gas dynamics (both outflow and inflow) towards both nuclei, only the eastern nucleus - which is the less luminous of the two, and which has not been suggested to host an AGN - exhibits enhanced CO luminosity relative to its FIR luminosity.

The large velocity dispersion found for the eastern nucleus is one possible source of energy for the warm CO emission. From Rangwala et al. (2011) (also, Kamenetzky et al. 2014), the total CO luminosity of Arp 220 is $L_{\mathrm{CO}} \approx 2 \times 10^{8} L_{\odot}$. We assume that half of this luminosity comes from the eastern nucleus. However, at the warm temperature inferred for this component, most of the gas cooling will be from molecular hydrogen, not CO (Neufeld et al. 1995). From Le Bourlot et al. (1999), the $\mathrm{H}_{2}$ cooling function is $\Lambda_{\mathrm{H}_{2}} \approx 10^{-22} \mathrm{erg} \mathrm{s}^{-1}(=11$ $\left.\mathrm{L}_{\odot} / \mathrm{M}_{\odot}\right)$ with a variation of about a factor of three over the range of temperatures and densities that characterize the warm molecular gas in Arp 220. With a warm gas mass $M_{\mathrm{H}_{2}} \simeq 1.3 \times 10^{8} M_{\odot}$ for the eastern nucleus (Table $1)$, the total warm gas cooling $L_{\text {warm }} \sim 6.5 \times 10^{42} \mathrm{erg}$ $\mathrm{s}^{-1}$ (with a range of a factor of 3 ). Comparison with equation 16] shows the energy dissipation rate is of the right order of magnitude to excite the warm molecular emission. This new result disagrees with the conclusions of Rangwala et al (2011). Their much smaller estimate of the energy available from turbulent heating was based on a gas velocity gradient derived from modeling of the global CO emission, while our new value uses the directly observed velocity dispersion provided by our ALMA observations.

We also consider the energy available from supernovae and stellar winds. Using the observed supernovae rate of $\nu_{\mathrm{SN}}=4 \pm 2$ per year (Lonsdale et al. 2006) and $\left(E_{\mathrm{SN}}=10^{51} \mathrm{erg}\right)$ in the following expression from Maloney (1999):

$$
L_{\mathrm{SN}} \sim 3 \times 10^{43}\left(\frac{\nu_{\mathrm{SN}}}{1 \mathrm{yr}^{-1}}\right)\left(\frac{E_{\mathrm{SN}}}{10^{51} \mathrm{ergs}}\right) \mathrm{erg} \mathrm{s}^{-1},
$$

we obtain a total mechanical energy of $\sim 10^{44} \mathrm{erg} \mathrm{s}^{-1}$. The energy output from stellar winds is similar to supernovae (McCray \& Kafatos 1987), giving a total of $\sim 2 \times 10^{44} \mathrm{erg} \mathrm{s}^{-1}$, also large enough to power the warm gas emission and maintain the observed velocity dispersion; this is consistent with the conclusions of Rangwala et al. (2011).

\section{HIGHLY EXCITED SIO ABSORPTION TOWARDS THE WESTERN NUCLEUS}

Absorption from the $\mathrm{SiO} J=16-15$ line at 694.293 $\mathrm{GHz}$ is seen towards the location of the continuum emission from the western nucleus (Figure 2(e) and Figure 3). The absorption is point-like with a full velocity width of at least $400 \mathrm{~km} \mathrm{~s}^{-1}$; the line width could be as large as $500 \mathrm{~km} \mathrm{~s}^{-1}$ if the $\mathrm{SiO}$ absorption continues through the small gap in our spectral coverage. The mean depth of the absorption in the central $200 \mathrm{~km} \mathrm{~s}^{-1}$ of the line is $-0.23 \mathrm{Jy} \mathrm{beam}^{-1}$ or $-6.9 \mathrm{~K}$. No significant $\mathrm{SiO}$ absorption is seen towards the eastern nucleus; any absorption in this nucleus must be at least 12 times fainter over the same relative velocity range.

The $\mathrm{SiO} J=5-4$ line at $217 \mathrm{GHz}$ has been previously seen in Arp 220 in emission (Martín et al. 2011), although the angular resolution of the data was insufficient to distinguish between the two nuclei. Martín et al. (2011) note that the $\mathrm{SiO}$ emission is not particularly enhanced in Arp 220 compared to other species. In contrast, García-Burillo et al. (2010) find that $\mathrm{SiO}$ emission near the AGN in NGC 1068 has an enhanced abundance by a factor of 10-100 compared to locations in the starburst ring. The fact that Martín et al. (2011) do not see an enhanced $\mathrm{SiO}$ abundance may be related to the inability of their data to isolate the region nearest to the AGN from surrounding starburst regions.

For Galactic star forming regions, Schilke et al. (1997) showed that $\mathrm{SiO}$ is released via neutral particles colliding with charged grains. Shock speeds of $25 \mathrm{~km} \mathrm{~s}^{-1}$ and densities of $1 \times 10^{5} \mathrm{~cm}^{-3}$ are sufficient to reproduce the observed $\mathrm{SiO}$ column densities. The mean density of the dusty disk in the western nucleus of Arp 220 is estimated to be $\gtrsim 10^{5} \mathrm{~cm}^{-3}$ (Paper I) and high turbulent velocities would be consistent with the large CO line-widths seen towards this nucleus. Thus, we might expect significant $\mathrm{SiO}$ to be present throughout this dense dusty disk. However, the $\mathrm{SiO} \mathrm{J}=16-15$ absorption is clearly more compact than the dusty disk; the strength of the $\mathrm{SiO}$ absorption does not change as the beam is increased to a FWHM of $0.5^{\prime \prime}$, while the peak continuum flux increases from 1.15 to $1.69 \mathrm{Jy} \mathrm{beam}^{-1}$. Thus, high excitation $\mathrm{SiO}$ molecules appear to be concentrated towards the center of the western nucleus. However, because the dust emission is quite optically thick $(\tau \sim 5$, Wilson et al. 2014), the radius of the region producing the $\mathrm{SiO}$ absorption has to be comparable to the scale height of the disk.

The question arises as to how $\mathrm{SiO}$ is excited to such a high energy level in Arp 220. The upper state of the SiO $J=16-15$ line is $283 \mathrm{~K}$ above ground and the transition has an Einstein coefficient $\log A_{i j}=-1.74$ (taken from the Splatalogue molecular database). Thus, the critical density of this transition is $\sim 2 \times 10^{8} \mathrm{~cm}^{-3}, 1000$ times higher than that of the CO $J=6-5$ line. Because the $\mathrm{SiO} J=16-15$ line is seen in absorption, the excitation temperature of this transition must be $<100-200 \mathrm{~K}$, the estimated brightness temperature of the continuum source at this frequency. One possible mechanism to excite $\mathrm{SiO}$ is via radiative pumping. Radiative pumping 
has been invoked to explain the high-J absorption lines of HCN seen in Arp 220 (Rangwala et al. 2011). The first excited vibrational state of $\mathrm{SiO}$ is at $\sim 8 \mu \mathrm{m}$, near the peak of a $360 \mathrm{~K}$ blackbody spectrum. Given the high mean temperature of the western nucleus, radiative pumping by the intense radiation field of an AGN or an extreme nuclear starburst appears to be a viable mechanism.

The alternative would be collisional excitation in the inner, presumably higher density regions, of the nuclear disk. However, a 50 pc radius disk with a mean density of $4 \times 10^{5} \mathrm{~cm}^{-3}$ requires a steep density profile $\left(\sim r^{-1}\right)$ to achieve densities comparable to the critical density before the dust reaches its sublimation temperature. Therefore, it is difficult to see how a sufficiently extended region of excited $\mathrm{SiO}$ could be achieved under these conditions.

\section{CONCLUSIONS}

Our Cycle-0 ALMA observations of the CO $J=6-5$ line show complex features in the morphology and kinematics of the warm molecular gas not seen before, neither in low- $J$ lines tracing cold gas nor in lower-resolution $\mathrm{CO}$ $J=6-5$ observations from the SMA. The overall morphology of the warm emission is similar to that traced by the low- $J$ lines. However, we detect an apparent offset of $\sim 100 \mathrm{pc}$ between the peak of the continuum emission and the peak of the line emission. This offset is present in both nuclei and is in the same direction. Comparison with kinematic models suggests that the offset arises due to the absorption of the entire South (blue) side of the central region containing the two nuclei by gas lying in front of the nuclei. There is also clear evidence in our data for redshifted absorption, which could be interpreted as an inflowing molecular filament. If a single coherent feature is responsible for all of the absorption features - both the redshifted absorption and the blueshifted absorption that produces the apparent offset and the line profile asymmetries - it has a total velocity extent of $\sim 700 \mathrm{~km} \mathrm{~s}^{-1}$ and a spatial extent of $\sim 400$ pc. Alternatively, the blueshifted absorption may arise in a larger (kpc)-scale molecular disk, as suggested by earlier interferometric observations, and have no dynamical connection with the infalling gas. Although similar asymmetries in the line profiles can be produced by a combination of rotation plus outflow rather than rotation plus absorption, on balance we believe that the data favor absorption rather than outflow as the source of the asymmetries.

From our modeling, we conclude that the dynamical center of the eastern nuclear disk is at the location of the continuum peak, and that a large gradient in the excitation is needed to explain the deep absorption detected at the center of the eastern nucleus. In addition, the velocity dispersion in the disk must be a significant fraction of the rotation velocity to be consistent with the large line widths observed in the two nuclei. The dynamical mass estimated from our kinematic model implies a very large mass surface density for the eastern nucleus, suggesting the disk could be gravitationally unstable and prone to disruption. However, the estimated Toomre $Q$-parameter for the gas disk of the eastern nucleus is (marginally) greater than unity, suggesting that the disk is plausibly stabilized against gravitational fragmentation and collapse by the large velocity dispersion.
We measure an unusually high ratio of $\mathrm{CO} J=6-5$ luminosity to the total FIR luminosity in the eastern nucleus compared to the western nucleus; for the latter the value is consistent with the average found in nearby galaxies. This suggests that there is an additional energy source, such as mechanical energy from shocks, present in the eastern nucleus that is generating more CO luminosity without affecting the dust continuum emission and hence the total FIR luminosity. We find that the total warm gas cooling from $\mathrm{H}_{2}$ approximately matches the energy dissipation rate (from velocity dispersion), suggesting that this energy dissipation could also potentially power the warm molecular emission, in addition to the energy from supernovae and stellar winds.

Without the exceptional sensitivity and spatial/spectral resolution from ALMA it would have not been possible to detect the complexity in the morphology and kinematics of the warm molecular gas in Arp 220, and to measure the various quantities associated with them with high signal-to-noise ratios. This is especially important for detecting the signature of inflowing molecular gas, which is much harder to detect. The high $\mathrm{S} / \mathrm{N}$ of finely sampled velocity profiles allowed us to distinguish between various kinematic models. It will be highly beneficial to have the same quality of observations for the multiple $\mathrm{CO}$ lines that are accessible with ALMA, to look for spatial variations in the gas excitation and better characterize the mass and excitation of the large-scale gas dynamics (both outflow and inflow) towards the two nuclei. This kind of spatial/kinematic analysis is highly complementary to the analysis of Herschel observations described in $\S 1$. Our accepted Cycle-2 observations of Arp 220 (and NGC 6240) will provide a step forward in this direction.

The National Radio Astronomy Observatory is a facility of the National Science Foundation operated under cooperative agreement by Associated Universities, Inc. This paper makes use of the following ALMA data: ADS/JAO.ALMA2011.0.00403.S. ALMA is a partnership of ESO (representing its member states), NSF (USA) and NINS (Japan), together with NRC (Canada) and NSC and ASIAA (Taiwan), in cooperation with the Republic of Chile. The Joint ALMA Observatory is operated by ESO, AUI/NRAO and NAOJ. We are very grateful to Adam Leroy for doing a custom reduction for our observations and providing very useful recommendations. We thank Kazushi Sakamoto for sharing his CO $J=3-2$ data cube observed by the Submillimeter Array. The research of Naseem Rangwala is supported by NASA ROSES grant NNX13AL16G. The research of Philip R. Maloney is supported by NASA grant 1487846 . The research of Christine D. Wilson (C.D.W.) is supported by grants from the Natural Sciences and Engineering Research Council of Canada. C.D.W. also thanks the European Southern Observatory and the National Radio Astronomy Observatory for visitor support. The research of Jason Glenn is supported by NASA grant 1472566 . 
APPENDIX

APPENDIX A: ADDITIONAL MODELING RESULTS
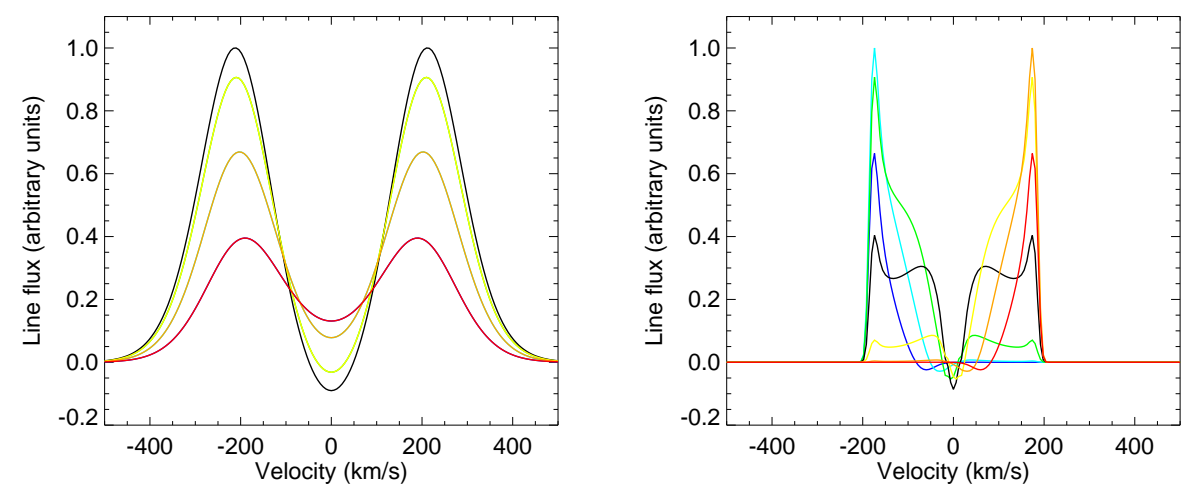

Figure 14. Velocity profiles generated from an axisymmetric disk model for two cases: (Top) Doppler parameter $(\sqrt{2} \times$ dispersion) $=$ $180 \mathrm{~km} \mathrm{~s}^{-1}$ and $\mathrm{V} \max =0 \mathrm{~km} \mathrm{~s}^{-1}$ and (Bottom) Doppler Parameter $=10 \mathrm{~km} \mathrm{~s}^{-1}$ and Vmax $=180 \mathrm{~km} \mathrm{~s}^{-1}$. Color coding as in Figure 7
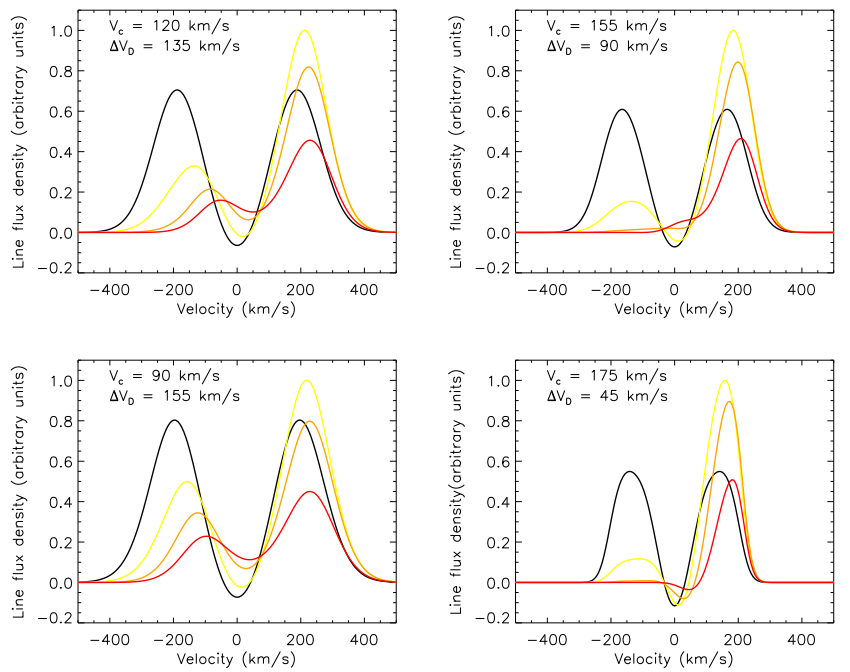

Figure 15. Model profiles for different values of Doppler parameter and maximum rotation velocity with their quadrature sum fixed. Color coding as in Figure 7

The strength of the emission on the opposite-velocity side of the absorption dip as one moves away from the center along the major axis towards either the red or blue sides of the disk, the relative peak heights along the major axis, and the shapes and velocity extents of the red and blue line profiles, can be used as diagnostics of the relative magnitudes of the velocity dispersion and rotation velocity. Since there are clearly systematic issues with the blue side of the disk, discussed in Section 4, we mostly consider the constraints provided by the red side of the emission. In the limit of zero rotation velocity (assuming the same optical depth and source function parameters as in Figure 7), the line profile is symmetric about the absorption dip, and the line peak flux densities are greatest at the disk center, while if the velocity dispersion is much smaller than the rotation velocity, the line profiles are very non-Gaussian, and peak sharply at the maximum rotation velocity at all positions along the major axis (see Figure 14). To illustrate the approximate allowed ranges of the rotation velocity and velocity dispersion, in Figure [15] we plot the resulting line profiles (at half-beamwidth intervals and Gaussian-weighted, as in Figures 7) for four different models, each labeled by $V_{c}$ and $\Delta V_{D}$. In all cases $V_{c}$ and $\Delta V_{D}$ have been varied so that their quadrature sum is nearly fixed, which ensures that the peak-to-peak separation of the red and blue peaks is similar to the observed $\left(\sim 350 \mathrm{~km} \mathrm{~s}^{-1}\right)$ in all models. Note that this separation is generally significantly larger than twice the rotation velocity, as is the extent of the emission in velocity space: this is a consequence of both the substantial velocity dispersion and of optical depth effects, as the models are not optically thin. For clarity, we only plot the models for the center and red-side positions; the blue-side emission lines are the mirror-image of the red-side. 
There is little change in the peak flux densities as $\Delta V_{D}$ is increased to $135 \mathrm{~km} \mathrm{~s}^{-1}$; the main change is a modest increase in the line widths. As is evident in Figure 7, the model line profiles are a little narrower than the observed, and do not exhibit the trend of increasing linewidth with distance along the major axis seen at the $\theta_{B}$ and $1.5 \theta_{B}$ offset points, indicating deviations from the model assumptions (constant $V_{c}$ and $\Delta V_{D}$ ). It does predict a slightly stronger red peak at $\Delta \theta=-\theta_{B} / 2$ (the blue side of the major axis) than the $\Delta V_{D}=120 \mathrm{~km} \mathrm{~s}^{-1}$ model, which is already slightly too strong compared to the observations.

Raising the rotation velocity to $155 \mathrm{~km} \mathrm{~s}^{-1}$ makes the lines narrower, slightly reduces the difference in peak flux density between the center and $\Delta \theta=3 \theta_{B} / 2$ positions, and substantially reduces the intensity of the blue-shifted emission. Reducing $V_{c}$ to $90 \mathrm{~km} \mathrm{~s}^{-1}$ and raising $\Delta V_{D}$ correspondingly (i.e., swapping the values from the previous model) makes the center and $\Delta \theta=\theta_{B}$ peak flux densities nearly equal, in substantial disagreement with the observations, and makes the blue-shifted emission substantially brighter. Finally, raising $V_{c}$ as high as $175 \mathrm{~km} \mathrm{~s}^{-1}$ produces nearly equal peak flux densities in the center and $\Delta \theta=3 \theta_{B} / 2$ positions, and more nearly equal peak flux densities in the $\Delta \theta=\theta_{B} / 2$ and $\Delta \theta=\theta_{B} /$ positions, again in disagreement with the observations.

In summary, these model variations suggest that the disk rotation velocity is unlikely to be substantially below 120 $\mathrm{km} \mathrm{s}^{-1}$, or above $155 \mathrm{~km} \mathrm{~s}^{-1}$, with the velocity dispersion scaled accordingly.

\section{REFERENCES}

Barcos-Muñoz, L., Leroy, A. K., Evans, A. S., Privon, G. C., Armus, L., Condon, J., Mazzarella, J. M., Meier, D. S., Momjian, E., Murphy, E. J., Ott, J., Reichardt, A., Sakamoto, K., Sanders, D. B., Schinnerer, E., Stierwalt, S., Surace, J. A., Thompson, T. A., \& Walter, F. 2014, ArXiv e-prints

Downes, D., \& Eckart, A. 2007, A\&A, 468, L57

Engel, H., Davies, R. I., Genzel, R., Tacconi, L. J., Sturm, E., \& Downes, D. 2011, ApJ, 729:58, 14pp

Etxaluze, M., Goicoechea, J. R., Cernicharo, J., Polehampton, E. T., Noriega-Crespo, A., Molinari, S., Swinyard, B. M., Wu, R., \& Bally, J. 2013, A\&A, 556, A137

García-Burillo, S., Usero, A., Fuente, A., Martín-Pintado, J., Boone, F., Aalto, S., Krips, M., Neri, R., Schinnerer, E., \& Tacconi, L. J. 2010, A\&A, 519, A2

Goicoechea, J. R., Etxaluze, M., Cernicharo, J., Gerin, M., Neufeld, D. A., Contursi, A., Bell, T. A., De Luca, M., Encrenaz, P., Indriolo, N., Lis, D. C., Polehampton, E. T., \& Sonnentrucker, P. 2013, ApJ, 769:L13, 7pp

Hummer, D. G., \& Rybicki, G. B. 1968, ApJ, 153, L107

Kamenetzky, J., Glenn, J., Rangwala, N., Maloney, P., Bradford, M., Wilson, C. D., Bendo, G. J., Baes, M., Boselli, A., Cooray, A., Isaak, K. G., Lebouteiller, V., Madden, S., Panuzzo, P., Schirm, M. R. P., Spinoglio, L., \& Wu, R. 2012, ApJ, 753:70, 17pp

Kamenetzky, J., Rangwala, N., Glenn, J., Maloney, P. R., \& Conley, A. 2014, ArXiv e-prints

Le Bourlot, J., Pineau des Forêts, G., \& Flower, D. R. 1999, MNRAS, 305, 802

Lonsdale, C. J., Diamond, P. J., Thrall, H., Smith, H. E., \& Lonsdale, C. J. 2006, ApJ, 647,185

Mac Low, M.-M. 1999, ApJ, 524, 169

Maloney, P. R. 1999, Ap\&SS, 266, 207

Martín, S., Krips, M., Martín-Pintado, J., Aalto, S., Zhao, J.-H., Peck, A. B., Petitpas, G. R., Monje, R., Greve, T. R., \& An, T. 2011, A\&A, 527, A36

Matsushita, S., Iono, D., Petitpas, G. R., Chou, R. C.-Y., Gurwell, M. A., Hunter, T. R., Muller, J. L. S., Peck, A. B., Sakamoto, K., Sawada Satoh, S., Wiedner, M. C., Wilner, D. J., \& Wilson, C. D. 2009, ApJ, 693, 56

McCray, R., \& Kafatos, M. 1987, ApJ, 317, 190

Meijerink, R., Kristensen, L. E., Weiß, A., van der Werf, P. P., Walter, F., Spaans, M., Loenen, A. F., Fischer, J., Israel, F. P., Isaak, K., Papadopoulos, P. P., Aalto, S., Armus, L., Charmandaris, V., Dasyra, K. M., Diaz-Santos, T., Evans, A., Gao, Y., González-Alfonso, E., Güsten, R., Henkel, C., Kramer, C., Lord, S., Martín-Pintado, J., Naylor, D., Sanders, D. B., Smith, H., Spinoglio, L., Stacey, G., Veilleux, S., \& Wiedner, M. C. 2013, ApJ, 762, L16

Neufeld, D. A., Lepp, S., \& Melnick, G. J. 1995, ApJS, 100, 132

Panuzzo, P., Rangwala, N., Rykala, A., Isaak, K. G., Glenn, J., Wilson, C. D., Auld, R., Baes, M., Barlow, M. J., Bendo, G. J., Bock, J. J., Boselli, A., Bradford, M., Buat, V., Castro-Rodríguez, N., Chanial, P., Charlot, S., Ciesla, L., Clements, D. L., Cooray, A., Cormier, D., Cortese, L., Davies, J. I., Dwek, E., Eales, S. A., Elbaz, D., Fulton, T., Galametz, M., Galliano, F., Gear, W. K., Gomez, H. L., Griffin, M., Hony, S., Levenson, L. R., Lu, N., Madden, S., O’Halloran, B., Okumura, K., Oliver, S., Page, M. J., Papageorgiou, A., Parkin, T. J., Pérez-Fournon, I., Pohlen, M., Polehampton, E. T., Rigby, E. E., Roussel, H., Sacchi, N., Sauvage, M., Schulz, B., Schirm, M. R. P., Smith, M. W. L., Spinoglio, L., Stevens, J. A., Srinivasan, S., Symeonidis, M., Swinyard, B., Trichas, M., Vaccari, M., Vigroux, L., Wozniak, H., Wright, G. S., \& Zeilinger, W. W. 2010, A\&A, 518, L37+

Papadopoulos, P. P., Zhang, Z.-Y., Xilouris, E. M., Weiss, A., van der Werf, P., Israel, F. P., Greve, T. R., Isaak, K. G., \& Gao, Y. 2014, ApJ, 788, 153

Pereira-Santaella, M., Spinoglio, L., Busquet, G., Wilson, C. D., Glenn, J., Isaak, K. G., Kamenetzky, J., Rangwala, N., Schirm, M. R. P., Baes, M., Barlow, M. J., Boselli, A., Cooray, A., \& Cormier, D. 2013, ApJ, 768, 55

Rangwala, N., Maloney, P. R., Glenn, J., Wilson, C. D., Rykala, A., Isaak, K., Baes, M., Bendo, G. J., Boselli, A., Bradford, C. M., Clements, D. L., Cooray, A., Fulton, T., Imhof, P., Kamenetzky, J., Madden, S. C., Mentuch, E., Sacchi, N., Sauvage, M., Schirm, M. R. P., Smith, M. W. L., Spinoglio, L., \& Wolfire, M. 2011, ApJ, 743:94, 19pp

Sakamoto, K., Aalto, S., Wilner, D. J., Black, J. H., Conway, J. E., Costagliola, F., Peck, A. B., Spaans, M., Wang, J., \& Wiedner, M. C. 2009, ApJ, 700, L104

Sakamoto, K., Scoville, N. Z., Yun, M. S., Crosas, M., Genzel, R., \& Tacconi, L. J. 1999, ApJ, 514, 68

Sakamoto, K., Wang, J., Wiedner, M. C., Wang, Z., Peck, A. B., Zhang, Q., Petitpas, G. R., Ho, P. T. P., \& Wilner, D. J. 2008, ApJ, 684, 957

Schilke, P., Walmsley, C. M., Pineau des Forets, G., \& Flower, D. R. 1997, A\&A, 321,293

Scoville, N. Z., Yun, M. S., \& Bryant, P. M. 1997, ApJ, 484, 702

Spinoglio, L., Pereira-Santaella, M., Busquet, G., Schirm, M. R. P., Wilson, C. D., Glenn, J., Kamenetzky, J., Rangwala, N., Maloney,

P. R., Parkin, T. J., Bendo, G. J., Madden, S. C., Wolfire, M. G., Boselli, A., Cooray, A., \& Page, M. J. 2012, ApJ, 758:108, 15pp

Toomre, A. 1964, ApJ, 139, 1217

van der Tak, F. F. S., Black, J. H., Schöier, F. L., Jansen, D. J., \& van Dishoeck, E. F. 2007, A\&A, 468, 627

Wilson, C. D., Rangwala, N., Glenn, J., Maloney, P. R., Spinoglio, L., \& Pereira-Santaella, M. 2014, ApJ, 789, L36 